\title{
ON OPTIMAL CONTROL PROBLEMS WITH CONTROLS APPEARING NONLINEARLY IN AN ELLIPTIC STATE EQUATION*
}

\author{
EDUARDO $\mathrm{CASAS}^{\dagger}$ AND FREDI TRÖLTZSCH ${ }^{\ddagger}$
}

\begin{abstract}
An optimal control problem for a semilinear elliptic equation is discussed, where the control appears nonlinearly in the state equation but is not included in the objective functional. The existence of optimal controls is proved by a measurable selection technique. First-order necessary optimality conditions are derived and two types of second-order sufficient optimality conditions are established. A first theorem invokes a well-known assumption on the set of zeros of the switching function. A second relies on coercivity of the second derivative of the reduced objective functional. The results are applied to the convergence of optimal state functions for a finite element discretizion of the control problem.
\end{abstract}

Key words. optimal control, elliptic equation, existence of optimal solutions, measurable selection, first- and second-order optimality conditions, convergence of numerical approximations

AMS subject classifications. 35J61, 49J20, 49K20, 49M25

DOI. $10.1137 / 19 \mathrm{M} 1293442$

1. Introduction. In this paper, we consider the following optimal control problem:

$$
\text { (P) } \min _{u \in \mathcal{U}_{a d}} J(u),
$$

where

$$
\mathcal{U}_{a d}=\left\{u \in L^{\infty}(\Omega): \alpha \leq u(x) \leq \beta \text { for a.a. } x \in \Omega\right\}
$$

with $-\infty<\alpha<\beta<+\infty$, and

$$
J(u)=\int_{\Omega} L\left(x, y_{u}(x)\right) d x .
$$

Above $L: \Omega \times \mathbb{R} \longrightarrow \mathbb{R}$ is a given function, and $y_{u}$ is the solution of the following elliptic equation:

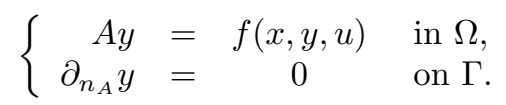

Precise assumptions on the data of the control problem $(\mathrm{P})$ will be given in section 2. However, it is important to mention here that $f$ is assumed to be monotone nonincreasing with respect to the variable $y$ so that (1.1) has a unique solution.

This paper has two main features: the control appears nonlinearly in the state equation and the objective functional only depends on the state. In mainstream papers, the control appears linearly in the state equation and often in a quadratic Tikhonov regularization term in the objective functional.

*Received by the editors October 15, 2019; accepted for publication (in revised form) May 26, 2020; published electronically July 14, 2020.

https://doi.org/10.1137/19M1293442

Funding: The first author was partially supported by the Spanish Ministerio de Economia y Competitividad under project MTM2017-83185-P.

${ }^{\dagger}$ Departmento de Matemática Aplicada y Ciencias de la Computación, E.T.S.I. Industriales y de Telecomunicación, Universidad de Cantabria, 39005 Santander, Spain (eduardo.casas@unican.es).

${ }^{\ddagger}$ Institut für Mathematik, Technische Universität Berlin, D-10623 Berlin, Germany (troeltzsch@ math.tu-berlin.de). 
Our setting has several consequences:

- The existence proof of optimal controls must differ from the standard one that is based on minimizing sequences of controls and their weak convergence. We will prove existence by a measurable selection theorem. In the framework of optimal control theory of ODEs similar techniques have been used. We refer, e.g., to the textbooks [19] and [13]. In PDE control, this issue has been addressed in [14], [15], or [21].

Using the mentioned measurable selection theorem, we are able to prove the convergence of a numerical approximation of the control problem. Indeed, in section 7 we consider a discretization of $(\mathrm{P})$ by finite elements. We prove the strong convergence in $H^{1}(\Omega)$ of optimal discrete states to associated ones of the original continuous problem. To our knowledge, this application of measurable selection theorems is new for the numerical approximation of problems like $(\mathrm{P})$.

- For optimality conditions, the superposition operator $u \mapsto f(\cdot, y, u)$ should be Fréchet-differentiable. In view of this, the only useful space that does not need strong growth conditions on $f$ is $L^{\infty}(\Omega)$. Then, however, the wellknown two-norm discrepancy is unavoidable in the discussion of second-order sufficient optimality conditions.

- Moreover, and this is another issue, second-order optimality conditions are delicate when the control is not explicitly included in the cost functional. Actually, the classical assumption $J^{\prime \prime}(\bar{u}) v^{2} \geq \delta\|v\|^{2} \forall v$ in the critical cone is not satisfied when $u$ appears linearly in the state equation; see, e.g., [24, Lemma 5.1]. Nevertheless, due to the fact that $f$ is nonlinear with respect to the control, the coercivity of $J^{\prime \prime}(\bar{u})$ can be fulfilled as we show in one example. This fact is crucial for some of our results on second-order conditions.

We will prove sufficient optimality conditions in two ways. In the first, we rely on the fact that bang-bang controls can be expected as optimal. This is due to the missing control in the objective. Here, by using a structural assumption on the optimal adjoint state, we show that the control satisfying the first-order optimality conditions is locally optimal in the sense of $L^{\infty}(\Omega)$. Additionally, a quadratic growth condition of $J$ can be deduced; see Theorem 5.3.

The second way is based on coercivity of $J^{\prime \prime}$ and a Legendre Clebsch condition on the Hamiltonian. Similar assumptions are known from ODE control. We refer to [20] and to the references therein. Under these hypotheses, we show local optimality of stationary controls in the sense of $L^{2}(\Omega)$, although the differentiability relies on the space $L^{\infty}(\Omega)$.

Summarizing, our paper contains the following main novelties. We show the existence of optimal controls by a measurable selection theorem. We prove different types of results on second-order sufficiency - one for bang-bang controls and one based on some hidden coercivity of $J^{\prime \prime}$, all for the case of controls appearing nonlinearly in the state equation. Finally, we discuss basic convergence properties for numerical discretizations of our problem.

2. Assumptions and preliminary results. In this paper we make the following assumptions.

Assumption $1 . \Omega$ is a bounded domain in $\mathbb{R}^{n}$ with $n=2$ or 3 having a Lipschitz boundary $\Gamma$. $A$ stands for the following partial differential operator in $\Omega$

$$
A y=-\sum_{i, j=1}^{n} \partial_{x_{j}}\left(a_{i j}(x) \partial_{x_{i}} y\right)+a_{0} y,
$$


where $a_{i j}, a_{0} \in L^{\infty}(\Omega), a_{0} \geq 0, a_{0} \not \equiv 0$, and

$$
\exists \Lambda>0 \text { such that } \sum_{i, j=1}^{n} a_{i j}(x) \xi_{i} \xi_{j} \geq \Lambda|\xi|^{2} \forall \xi \in \mathbb{R}^{n} \text { and for a.a. } x \in \Omega \text {. }
$$

By $\partial_{n_{A}} y$ we denote the boundary operator

$$
\partial_{n_{A}} y=\sum_{j=1}^{n}\left[\sum_{i=1}^{n} a_{i j}(x) \partial_{x_{i}} y_{i}\right] n_{j},
$$

where $n(x)=\left(n_{j}(x)\right)_{j=1}^{n}$ is the unit outward normal vector to $\Gamma$ at the point $x \in \Gamma$. In what follows, we denote with $A^{*}$ the adjoint differential operator of $A$,

$$
A^{*} \varphi=-\sum_{i, j=1}^{n} \partial_{x_{j}}\left(a_{j i}(x) \partial_{x_{i}} \varphi\right)+a_{0} \varphi .
$$

Assumption 2. We suppose that $f: \Omega \times \mathbb{R}^{2} \longrightarrow \mathbb{R}$ is a Carathéodory function of class $C^{2}$ with respect to the last two components satisfying

- $f(\cdot, 0,0) \in L^{\bar{p}}(\Omega)$ for some $\bar{p}>\frac{n}{2}$ and

$$
\frac{\partial f}{\partial y}(x, y, u) \leq 0 \text { a.e. in } \Omega \text { and } \forall(y, u) \in \mathbb{R}^{2},
$$

- $\forall M>0 \quad \exists C_{f, M}$ such that

$$
\sum_{1 \leq i+j \leq 2}\left|\frac{\partial^{i+j} f}{\partial y^{i} \partial u^{j}}(x, y, u)\right| \leq C_{f, M}
$$

for a.a. $x \in \Omega$ and $\forall(y, u) \in \mathbb{R}^{2}$ with $|y| \leq M$ and $u \in[\alpha, \beta]$,

- $\forall \varepsilon>0$ and $\forall M>0 \quad \exists \rho>0$ such that, if $\left|y_{1}\right|,\left|y_{2}\right| \leq M,\left|y_{1}-y_{2}\right|<\rho$, $u_{i} \in[\alpha, \beta],\left|u_{1}-u_{2}\right|<\rho$, then

$$
\sum_{i+j=2}\left|\frac{\partial^{i+j} f}{\partial y^{i} \partial u^{j}}\left(x, y_{2}, u_{2}\right)-\frac{\partial^{i+j} f}{\partial y^{i} \partial u^{j}}\left(x, y_{1}, u_{1}\right)\right|<\varepsilon \text { for a.a. } x \in \Omega .
$$

Assumption 3 . We suppose that $L: \Omega \times \mathbb{R} \longrightarrow \mathbb{R}$ is a Carathéodory function of class $C^{2}$ with respect to the second component that satisfies

- $(2.4)$

$$
L(\cdot, 0) \in L^{1}(\Omega)
$$

- $\forall M>0 \exists \psi_{M} \in L^{\bar{p}}(\Omega)$ and $\exists C_{L, M}>0$ such that

$$
\left|\frac{\partial L}{\partial y}(x, y)\right| \leq \psi_{M}(x) \text { and }\left|\frac{\partial^{2} L}{\partial y^{2}}(x, y)\right| \leq C_{L, M} \forall|y| \leq M \text { and a.a. } x \in \Omega,
$$

- $\forall \varepsilon>0$ and $\forall M>0 \exists \rho>0$ such that, if $\left|y_{1}\right|,\left|y_{2}\right| \leq M$ and $\left|y_{1}-y_{2}\right|<\rho$,

$$
\left|\frac{\partial^{2} L}{\partial y^{2}}\left(x, y_{2}\right)-\frac{\partial^{2} L}{\partial y^{2}}\left(x, y_{1}\right)\right|<\varepsilon \text { for a.a. } x \in \Omega .
$$

Copyright $@$ by SIAM. Unauthorized reproduction of this article is prohibited. 
These assumptions are obviously satisfied by the classical tracking cost functional $L(x, y)=\frac{1}{2}\left(y-y_{d}(x)\right)^{2}$ provided that $y_{d} \in L^{2}(\Omega)$.

TheOREm 2.1. Suppose that Assumptions 1 and 2 hold. Then, for every $u \in$ $L^{\infty}(\Omega)$, the state equation (1.1) has a unique solution $y_{u} \in H^{1}(\Omega) \cap C^{0, \mu}(\bar{\Omega})$ for some $\mu \in(0,1]$ independent of $u$. Moreover, with some constant $C_{u}$, we have the estimate

$$
\left\|y_{u}\right\|_{H^{1}(\Omega)}+\left\|y_{u}\right\|_{C^{0, \mu}(\bar{\Omega})} \leq C_{u}\left(\|f(\cdot, 0,0)\|_{L^{\bar{p}}(\Omega)}+1\right),
$$

where $C_{u}$ depends on $\|u\|_{L^{\infty}(\Omega)}$. Further, there exists a constant $C_{\alpha, \beta}$ such that

$$
\left\|y_{u}\right\|_{H^{1}(\Omega)}+\left\|y_{u}\right\|_{C^{0, \mu}(\bar{\Omega})} \leq C_{\alpha, \beta}\left(\|f(\cdot, 0,0)\|_{L^{\bar{p}}(\Omega)}+1\right) \quad \forall u \in \mathcal{U}_{a d} .
$$

Finally, if $\left\{u_{k}\right\}_{k=1}^{\infty}$ is a bounded sequence in $L^{\infty}(\Omega)$ converging to $u$ in $L^{1}(\Omega)$, then

$$
\lim _{k \rightarrow \infty}\left(\left\|y_{u_{k}}-y_{u}\right\|_{H^{1}(\Omega)}+\left\|y_{u_{k}}-y_{u}\right\|_{C^{0, \mu}(\bar{\Omega})}\right)=0 .
$$

Proof. Due to the monotonicity of $f$ with respect to $y$, the existence of a unique solution of (1.1) in $H^{1}(\Omega) \cap L^{\infty}(\Omega)$ is a classical result; see, for instance, [3] or [25, Theorem 4.7]. In these references, the estimate

$$
\left\|y_{u}\right\|_{L^{\infty}(\Omega)} \leq M_{1}\|f(\cdot, 0, u)\|_{L^{\bar{p}}(\Omega)}
$$

is proved with some constant $M_{1}>0$. Taking $M_{2}=\|u\|_{L^{\infty}(\Omega)}$ we infer from (2.2) and the mean value theorem

$$
|f(x, 0, u(x))| \leq|f(x, 0,0)|+\left|\frac{\partial f}{\partial u}(x, 0, \theta(x) u(x))\right||u(x)| \leq|f(x, 0,0)|+C_{f, M_{2}} M_{2},
$$

where $\theta: \Omega \longrightarrow[0,1]$ is a measurable function. Combining (2.10) and (2.11), and using again (2.2), we obtain with

$$
M=\max \left\{M_{2}, M_{1}\|f(\cdot, 0,0)\|_{L^{\bar{p}}(\Omega)}+M_{1} M_{2} C_{f, M_{2}}|\Omega|^{\frac{1}{\bar{p}}}\right\}
$$

the estimate

$$
\begin{aligned}
\left|f\left(x, y_{u}(x), u(x)\right)\right| \leq & |f(x, 0,0)|+\left|\frac{\partial f}{\partial y}\left(x, \vartheta(x) y_{u}, \vartheta(x) u(x)\right)\right|\left|y_{u}(x)\right| \\
& +\left|\frac{\partial f}{\partial u}\left(x, \vartheta(x) y_{u}, \vartheta(x) u(x)\right)\right||u(x)| \leq|f(x, 0,0)|+2 C_{f, M} M
\end{aligned}
$$

for some measurable function $\vartheta: \Omega \longrightarrow[0,1]$. Then the $C^{0, \mu}(\bar{\Omega})$ regularity for some $\mu \in(0,1]$ is proved in $[18$, section 3.14$]$ and the estimate $(2.7)$ holds. The inequality (2.8) is an immediate consequence of (2.7) and the boundedness of $\mathcal{U}_{a d}$ in $L^{\infty}(\Omega)$. It remains to prove (2.9). First, we observe that the boundedness of $\left\{u_{k}\right\}_{k=1}^{\infty}$ in $L^{\infty}(\Omega)$ and the strong convergence in $L^{1}(\Omega)$ imply that $u_{k} \rightarrow u$ in every space $L^{p}(\Omega)$ with $p<+\infty$. In particular, this convergence holds in $L^{\bar{p}}(\Omega)$. Moreover, from (2.7) we deduce the existence of a constant $M$ such that

$$
\left\|y_{u_{k}}\right\|_{L^{\infty}(\Omega)}+\left\|u_{k}\right\|_{L^{\infty}(\Omega)}+\left\|y_{u}\right\|_{L^{\infty}(\Omega)}+\|u\|_{L^{\infty}(\Omega)} \leq M \quad \forall k \geq 1 .
$$

Let $a: H^{1}(\Omega) \times H^{1}(\Omega) \longrightarrow \mathbb{R}$ be the variational form associated to the elliptic operator $A$. Subtracting the equations satisfied by $y_{u_{k}}$ and $y_{u}$, multiplying them by

Copyright $@$ by SIAM. Unauthorized reproduction of this article is prohibited. 
$y_{u_{k}}-y_{u}$, and integrating by parts, we get with Assumption 1, the monotonicity of $f$ with respect to $y$, and $(2.2)$

$$
\begin{aligned}
\Lambda \| & y_{u_{k}}-y_{u} \|_{H^{1}(\Omega)}^{2} \leq a\left(y_{u_{k}}-y_{u}, y_{u_{k}}-y_{u}\right) \\
= & \int_{\Omega}\left[f\left(x, y_{u_{k}}, u_{k}\right)-f\left(x, y_{u}, u\right)\right]\left(y_{u_{k}}-y_{u}\right) d x \\
= & \int_{\Omega}\left[f\left(x, y_{u_{k}}, u_{k}\right)-f\left(x, y_{u}, u_{k}\right)\right]\left(y_{u_{k}}-y_{u}\right) d x \\
& \quad+\int_{\Omega}\left[f\left(x, y_{u}, u_{k}\right)-f\left(x, y_{u}, u\right)\right]\left(y_{u_{k}}-y_{u}\right) d x \\
\leq & \int_{\Omega}\left[f\left(x, y_{u}, u_{k}\right)-f\left(x, y_{u}, u\right)\right]\left(y_{u_{k}}-y_{u}\right) d x \leq C_{f, M} \int_{\Omega}\left|u_{k}-u \| y_{u_{k}}-y_{u}\right| d x \\
\leq & C_{f, M}\left\|u_{k}-u\right\|_{L^{\bar{p}}(\Omega)}\left\|y_{u_{k}}-y_{u}\right\|_{L^{\bar{p}^{\prime}}(\Omega)} \leq C_{f, M} C_{\bar{p}, \Omega}\left\|u_{k}-u\right\|_{L^{\bar{p}}(\Omega)}\left\|y_{u_{k}}-y_{u}\right\|_{H^{1}(\Omega)}
\end{aligned}
$$

where we have used that $\bar{p}>\frac{n}{2}$ and consequently its conjugate satisfies $\bar{p}^{\prime}<\frac{n}{n-2}$. We also invoked the continuity of the embedding $H^{1}(\Omega) \subset L^{\frac{2 n}{n-2}}(\Omega) \subset L^{\bar{p}^{\prime}}(\Omega)$. The above estimate leads to

$$
\left\|y_{u_{k}}-y_{u}\right\|_{H^{1}(\Omega)} \leq \frac{C_{f, M} C_{\bar{p}, \Omega}}{\Lambda}\left\|u_{k}-u\right\|_{L^{\bar{p}}(\Omega)} \rightarrow 0, k \rightarrow \infty .
$$

Now, (2.7) implies that $\left\{y_{u_{k}}\right\}_{\underline{k}=1}^{\infty}$ is bounded in $C^{0, \mu}(\bar{\Omega})$. Hence, the compactness of the embedding $C^{0, \mu}(\bar{\Omega}) \subset C(\bar{\Omega})$ and the convergence $y_{u_{k}} \rightarrow y_{u}$ in $H^{1}(\Omega)$ imply that $y_{u_{k}} \rightarrow y_{u}$ in $C(\bar{\Omega}), k \rightarrow \infty$. Finally, taking into account that

$$
A\left(y_{u_{k}}-y_{u}\right)=f\left(x, y_{u_{k}}, u_{k}\right)-f\left(x, y_{u}, u\right)
$$

and using again the results in [18, section 3.14] along with (2.2) we obtain

$$
\begin{aligned}
\left\|y_{u_{k}}-y_{u}\right\|_{C^{0, \mu}(\bar{\Omega})} & \leq C\left\|f\left(x, y_{u_{k}}, u_{k}\right)-f\left(x, y_{u}, u\right)\right\|_{L^{\bar{p}}(\Omega)} \\
& \leq C C_{f, M}\left(\left\|y_{u_{k}}-y_{u}\right\|_{L^{\bar{p}}(\Omega)}+\left\|u_{k}-u\right\|_{L^{\bar{p}}(\Omega)}\right) \rightarrow 0 .
\end{aligned}
$$

In what follows, we will set $Y=H^{1}(\Omega) \cap C^{0, \mu}(\bar{\Omega})$ and denote by $G: L^{\infty}(\Omega) \longrightarrow Y$ the mapping associating to each control $u$ the corresponding state $y_{u}, G(u)=y_{u}$. This mapping is well defined according to the previous theorem. The next theorem establishes the differentiability of $G$.

ThEOREM 2.2. Under Assumptions 1 and 2, the mapping $G$ is of class $C^{2}$. For every $u, v, v_{1}, v_{2} \in L^{\infty}(\Omega), z_{v}=G^{\prime}(u) v$ and $z_{v_{1}, v_{2}}=G^{\prime \prime}(u)\left(v_{1}, v_{2}\right)$ are the solutions of the problems

$$
\left\{\begin{aligned}
A z & =\frac{\partial f}{\partial y}\left(x, y_{u}, u\right) z+\frac{\partial f}{\partial u}\left(x, y_{u}, u\right) v & & \text { in } \Omega, \\
\partial_{n_{A}} z & =0 & & \text { on } \Gamma,
\end{aligned}\right.
$$

and, respectively,

$$
\left\{\begin{array}{rlrl}
A z= & \frac{\partial f}{\partial y}\left(x, y_{u}, u\right) z+\frac{\partial^{2} f}{\partial y^{2}}\left(x, y_{u}, u\right) z_{v_{1}} z_{v_{2}} & \\
& +\frac{\partial^{2} f}{\partial y \partial u}\left(x, y_{u}, u\right)\left(z_{v_{1}} v_{2}+v_{1} z_{v_{2}}\right)+\frac{\partial^{2} f}{\partial u^{2}}\left(x, y_{u}, u\right) v_{1} v_{2} & & \text { in } \Omega \\
\partial_{n_{A}} z= & 0 & & \text { on } \Gamma
\end{array}\right.
$$

where $z_{v_{i}}=G^{\prime}(u) v_{i}, i=1,2$.

Copyright (C) by SIAM. Unauthorized reproduction of this article is prohibited. 
Proof. Let us consider the vector space

$$
X=\left\{y \in H^{1}(\Omega): A y \in L^{\bar{p}}(\Omega)\right\} .
$$

Endowed with the graph norm

$$
\|y\|_{X}=\|y\|_{H^{1}(\Omega)}+\|A y\|_{L^{\bar{p}}(\Omega)},
$$

$\left(X,\|\cdot\|_{X}\right)$ is a Banach space. If we take $q=\min \{2, \bar{p}\}$, then $\partial_{n_{A}}: X \longrightarrow W^{-\frac{1}{q}, q}(\Gamma)$ is a linear and continuous mapping; see [6].

Now, we define

$$
V=\left\{y \in Y: A y \in L^{\bar{p}}(\Omega) \text { and } \partial_{n_{A}} y=0 \text { on } \Gamma\right\}
$$

and take

$$
\|y\|_{V}=\|y\|_{Y}+\|A y\|_{L^{\bar{p}}(\Omega)} .
$$

Then $\left(V,\|\cdot\|_{V}\right)$ is a Banach space. We consider the mapping

$$
\mathcal{F}: V \times L^{\infty}(\Omega) \longrightarrow L^{\bar{p}}(\Omega), \quad \mathcal{F}(y, u)=A y-f(x, y, u) .
$$

It is easy to check with (2.2) that $x \mapsto f(x, y(x), u(x))$ is a function belonging to $L^{\bar{p}}(\Omega)$ for every $(y, u) \in V \times L^{\infty}(\Omega)$. Moreover, $\mathcal{F}$ is of class $C^{2}$. In addition, if $y_{u}=G(u)$, then $\mathcal{F}\left(y_{u}, u\right)=0$ holds. We also have that

$$
\frac{\partial \mathcal{F}}{\partial y}\left(y_{u}, u\right): V \longrightarrow L^{\bar{p}}(\Omega), \quad \frac{\partial \mathcal{F}}{\partial y}\left(y_{u}, u\right) z=A z-\frac{\partial f}{\partial y}\left(x, y_{u}, u\right) z
$$

is an isomorphism. Indeed, this is a consequence of the fact that the Neumann problem

$$
\left\{\begin{aligned}
A z-\frac{\partial f}{\partial y}\left(x, y_{u}, u\right) z & =v & & \text { in } \Omega, \\
\partial_{n_{A}} z & =0 & & \text { on } \Gamma
\end{aligned}\right.
$$

has a unique solution $z \in V$ depending continuously on $v \in L^{\bar{p}}(\Omega)$. Now, the theorem follows easily by applying the implicit function theorem.

Thanks to the chain rule, we deduce the differentiability of $J$ from the previous theorem.

TheOREm 2.3. Under Assumptions 1 and 2, the mapping $J: L^{\infty}(\Omega) \longrightarrow \mathbb{R}$ is of class $C^{2}$ and we have

$J^{\prime}(u) v=\int_{\Omega} \varphi_{u} \frac{\partial f}{\partial u}\left(x, y_{u}, u\right) v d x \quad \forall u, v \in L^{\infty}(\Omega)$,

$J^{\prime \prime}(u)\left(v_{1}, v_{2}\right)=\int_{\Omega} \frac{\partial^{2} L}{\partial y^{2}}\left(x, y_{u}\right) z_{v}^{2} d x$

$+\int_{\Omega} \varphi_{u}\left(\frac{\partial^{2} f}{\partial y^{2}}\left(x, y_{u}, u\right) z_{v_{1}} z_{v_{2}}+\frac{\partial^{2} f}{\partial y \partial u}\left(x, y_{u}, u\right)\left(v_{1} z_{v_{2}}+v_{2} z_{v_{1}}\right)+\frac{\partial^{2} f}{\partial u^{2}}\left(x, y_{u}, u\right) v_{1} v_{2}\right) d x$

$\forall u, v, v_{1}, v_{2} \in L^{\infty}(\Omega)$, where $z_{v}=G^{\prime}(u) v$ and $z_{v_{i}}=G^{\prime}(u) v_{i}, i=1,2$, and $\varphi_{u} \in$ $H^{1}(\Omega) \cap C^{0, \mu}(\bar{\Omega})$ is the adjoint state associated with $u$, defined as the solution of the adjoint equation

Copyright $@$ by SIAM. Unauthorized reproduction of this article is prohibited. 


$$
\left\{\begin{aligned}
A^{*} \varphi & =\frac{\partial f}{\partial y}\left(x, y_{u}, u\right) \varphi+\frac{\partial L}{\partial y}\left(x, y_{u}\right) & & \text { in } \Omega, \\
\partial_{n_{A}^{*}} \varphi & =0 & & \text { on } \Gamma .
\end{aligned}\right.
$$

We finish this section with the following observation: from (2.16) and (2.5) we deduce the existence of a constant $M^{*}$ such that

$$
\left\|\varphi_{u}\right\|_{H^{1}(\Omega)}+\left\|\varphi_{u}\right\|_{C^{0, \mu}(\bar{\Omega})} \leq M^{*} \quad \forall u \in \mathcal{U}_{a d} .
$$

3. Existence of optimal controls. If the control appears linearly in the state equation, the standard method for proving existence of an optimal control is as follows. An infimal sequence of controls is considered that is bounded. Then a weakly converging subsequence is selected that eventually converges to an optimal control. This method cannot in general be applied to controls appearing nonlinearly.

We will use a technique that is based on measurable selection theorems, in particular on the well-known Filippov theorem. This method was often applied to control problems with ordinary differential equations but rarely used for partial differential equations.

Let us start with some examples that introduce some specific difficulties with controls appearing nonlinearly.

Example 3.1. Our first example illustrates an important effect that is related to the nonlinear appearance of controls. We consider the following linear state equation with control $u \in L^{2}(\Omega)$ :

$$
\left\{\begin{aligned}
-\Delta y(x)+y(x) & =u(x)^{2} & & \text { in } \Omega, \\
\partial_{n} y & =0 & & \text { on } \Gamma .
\end{aligned}\right.
$$

Select a sequence of bang-bang control functions $u_{k} \in L^{\infty}(\Omega)$ such that

$$
u_{k}(x) \in\{-1,1\} \text { a.e. in } \Omega
$$

and $u_{k} \rightarrow 0$ (weakly) in $L^{2}(\Omega)$. It is easy to construct such a sequence.

Obviously, we have $u_{k}(x)^{2}=1 \forall k$, hence the associated states $y_{k}=y_{u_{k}}$ are $y_{k}(x)=1$ a.e. in $\Omega$. This stationary sequence converges uniformly to $y=1$, but this function $y$ is not the state associated with the weak limit control $\tilde{u}=0$.

The reader might object that the nonlinearity $u^{2}$ is too simple, since $u_{k}^{2} \equiv 1$ does not depend on $k$. Therefore, we discuss also a less naive example.

Example 3.2. Here, we consider the nonlinear equation

$$
\left\{\begin{aligned}
-\Delta y(x)+y(x) & =e^{-y(x)} u(x)^{2}+u(x) & & \text { in } \Omega, \\
\partial_{n} y & =0 & & \text { on } \Gamma,
\end{aligned}\right.
$$

where we insert the sequence $u_{k}$ defined in Example 3.1. The associated states $y_{k}$ solve the equation

$$
\left\{\begin{aligned}
-\Delta y_{k}(x)+y_{k}(x) & =e^{-y_{k}(x)}+u_{k}(x) & & \text { in } \Omega \\
\partial_{n} y_{k} & =0 & & \text { on } \Gamma .
\end{aligned}\right.
$$

The states $y_{k}$ converge strongly in $H^{1}(\Omega) \cap L^{\infty}(\Omega)$ to the solution $y$ of

$$
\left\{\begin{aligned}
-\Delta y(x)+y(x) & =e^{-y(x)} & & \text { in } \Omega, \\
\partial_{n} y & =0 & & \text { on } \Gamma,
\end{aligned}\right.
$$

but not to the state $y_{\tilde{u}}=0$ that is associated with the weak limit control $\tilde{u}=0$. 
TheOREm 3.3. Under Assumptions 1-3, the optimal control problem (P) has at least one solution.

Proof. Let $\left\{u_{k}\right\}_{k=1}^{\infty} \subset \mathcal{U}_{a d}$ be a minimizing sequence of (P): $J\left(u_{k}\right) \searrow \inf (\mathrm{P})$ as $k \rightarrow \infty$. From (2.8) we deduce that $\left\{y_{u_{k}}\right\}_{k=1}^{\infty}$ is bounded in $H^{1}(\Omega) \cap C^{0, \mu}(\bar{\Omega})$. Therefore, there exists a subsequence, denoted in the same way, and a function $\bar{y} \in$ $H^{1}(\Omega) \cap C(\bar{\Omega})$ such that $y_{u_{k}} \rightarrow \bar{y}$ in $H^{1}(\Omega)$ and $y_{u_{k}} \rightarrow \bar{y}$ in $C(\bar{\Omega})$. Moreover, $\bar{y}$ satisfies

$$
\int_{\Omega} L(x, \bar{y}(x)) d x=\lim _{k \rightarrow \infty} \int_{\Omega} L\left(x, y_{u_{k}}(x)\right) d x=\lim _{k \rightarrow \infty} J\left(u_{k}\right)=\inf (\mathrm{P}) .
$$

The first identity follows from Lebesgue's dominated convergence theorem. Indeed, taking $M=\max _{k \geq 1}\left\|y_{u_{k}}\right\|_{C(\bar{\Omega})}$ we deduce from (2.5) and the mean value theorem

$$
\left|L\left(x, y_{u_{k}}(x)\right)\right| \leq|L(x, 0)|+\left|\psi_{M}(x)\right| M \quad \text { for a.a. } x \in \Omega \text {. }
$$

Hence, we have with $(2.4)$ that $\left\{L\left(\cdot, y_{u_{k}}\right)\right\}_{k=1}^{\infty}$ is dominated by an $L^{1}(\Omega)$-function.

To conclude the proof, it is enough to show that $\bar{y}$ is the solution of (1.1) associated to some control $\bar{u} \in \mathcal{U}_{a d}$. Then, $\bar{u}$ is a solution of $(\mathrm{P})$. To this end, we introduce the multifunction

$$
F: \Omega \longrightarrow \mathcal{P}(\mathbb{R}), \quad F(x)=\{f(x, \bar{y}(x), s): s \in[\alpha, \beta]\} .
$$

Since $f$ is continuous respect to the last variable, we have that $F(x)$ is a closed and bounded interval of $\mathbb{R}$ for almost all $x \in \Omega$. Now, we define

$$
S=\left\{g \in L^{\bar{p}}(\Omega): g(x) \in F(x) \text { for a.a. } x \in \Omega\right\} .
$$

It is obvious that $S$ is a convex and closed subset of $L^{\bar{p}}(\Omega)$. Finally, setting $g_{k}(x)=$ $f\left(x, \bar{y}(x), u_{k}(x)\right)$, we have that $\left\{g_{k}\right\}_{k=1}^{\infty}$ is a sequence contained in $S$. Indeed, it is obvious that $g_{k}(x) \in F(x)$ for almost all $x \in \Omega$. Let us prove that every function $g_{k}$ belongs to $L^{\bar{p}}(\Omega)$. From $(2.8)$ we have that

$$
M=\|\bar{y}\|_{C(\bar{\Omega})}+\max \{|\alpha|,|\beta|\}<\infty
$$

holds. Then, from (2.2) and using the mean value theorem we infer that $\left|g_{k}(x)\right| \leq$ $|f(x, 0,0)|+C_{f, M} 2 M$, hence $\left\|g_{k}\right\|_{L^{\bar{p}}(\Omega)} \leq\|f(\cdot, 0,0)\|_{L^{\bar{p}}(\Omega)}+C_{f, M} 2 M|\Omega|^{\frac{1}{\bar{p}}}$. Thus, $\left\{g_{k}\right\}_{k=1}^{\infty}$ is a bounded sequence in $L^{\bar{p}}(\Omega)$. Therefore, we can extract a subsequence, denoted in the same form, such that $g_{k} \rightarrow \bar{g}$ in $L^{\bar{p}}(\Omega)$. Since $S$ is weakly closed in $L^{\bar{p}}(\Omega)$ we have that $\bar{g} \in S$. Now, from the classical Filippov theorem (see [16] or [19]), we deduce the existence of a measurable function $\bar{u}: \Omega \longrightarrow[\alpha, \beta]$, i.e., $\bar{u} \in \mathcal{U}_{a d}$, such that $\bar{g}(x)=f(x, \bar{y}(x), \bar{u}(x))$ for almost all $x \in \Omega$. We conclude the proof by showing that $\bar{y}=y_{\bar{u}}$. It is obvious that

$$
A y_{u_{k}}=g_{k}+\left[f\left(x, y_{u_{k}}, u_{k}\right)-f\left(x, \bar{y}, u_{k}\right)\right] \quad \text { in } \Omega .
$$

Using again (2.2) we get

$$
\left\|f\left(x, y_{u_{k}}, u_{k}\right)-f\left(x, \bar{y}, u_{k}\right)\right\|_{L^{\infty}(\Omega)} \leq C_{f, M}\left\|y_{u_{k}}-\bar{y}\right\|_{L^{\infty}(\Omega)} \rightarrow 0 \text {, when } k \rightarrow \infty .
$$

Hence, passing to the limit in the above equation, we obtain that $A \bar{y}=f(\cdot, \bar{y}, \bar{u})$ in $\Omega$, as desired. 
4. First- and second-order necessary optimality conditions. The goal of this section is to derive the first- and second-order necessary optimality conditions to be fulfilled by any local solution of $(\mathrm{P})$. Given $p \in[1, \infty]$, we say that $\bar{u}$ is a local minimum of $(\mathrm{P})$ in the $L^{p}(\Omega)$-sense if

$$
J(\bar{u}) \leq J(u) \quad \forall u \in \mathcal{U}_{a d} \cap B_{\varepsilon}(\bar{u}),
$$

where $B_{\varepsilon}(\bar{u})=\left\{u \in L^{p}(\Omega):\|u-\bar{u}\|_{L^{p}(\Omega)} \leq \varepsilon\right\}$. We say that $\bar{u}$ is a strict local minimum if the above inequality is strict whenever $u \neq \bar{u}$.

The Hamiltonian of $(\mathrm{P})$ is given by

$$
H: \Omega \times \mathbb{R}^{3} \longrightarrow \mathbb{R}, \quad H(x, y, \varphi, u)=L(x, y)+\varphi f(x, y, u) .
$$

Now we formulate the first-order necessary optimality conditions for $(\mathrm{P})$.

Theorem 4.1. Suppose that Assumptions 1-3 hold. Let $\bar{u}$ be a local minimum of (P) in the $L^{p}(\Omega)$-sense $(1 \leq p \leq \infty)$, then

$$
\int_{\Omega} \frac{\partial H}{\partial u}(x, \bar{y}, \bar{\varphi}, \bar{u})(u-\bar{u}) d x \geq 0 \quad \forall u \in \mathcal{U}_{a d} .
$$

Moreover, if $p<\infty$, then the Pontryagin principle holds,

$$
H(x, \bar{y}(x), \bar{\varphi}(x), \bar{u}(x))=\min _{s \in[\alpha, \beta]} H(x, \bar{y}(x), \bar{\varphi}(x), s) \quad \text { for a.a. } x \in \Omega .
$$

Proof. Due to the convexity of $\mathcal{U}_{a d}$, it is well known that any local solution $\bar{u}$ satisfies $J^{\prime}(\bar{u})(u-\bar{u}) \geq 0 \forall u \in \mathcal{U}_{a d}$. Then the inequality (4.1) follows from (2.14) and the definition of $H$.

Now we assume that $p<\infty$ and prove (4.2). Let $u \in \mathcal{U}_{a d}$ be a fixed control. We define $h(x)=f(x, \bar{y}(x), u(x))-f(x, \bar{y}(x), \bar{u}(x))$. From (2.1) and (2.2) we deduce that $h \in L^{\bar{p}}(\Omega)$. Let $\left\{v_{j}\right\}_{j=1}^{\infty}$ be a dense sequence in $L^{1}(\Omega)$. For every $k \geq 1$ we define the function $g_{k} \in L^{1}(\Omega)^{k+1}$ by $g_{k}=\left(1, v_{1}, \ldots, v_{k}\right)$. Given $\rho \in(0,1)$ arbitrarily, we deduce from Lyapunov's convexity theorem the existence of measurable sets $E_{\rho}^{k} \subset \Omega$ such that

$$
\int_{E_{\rho}^{k}} g_{k}(x) d x=\rho \int_{\Omega} g_{k}(x) d x \quad \forall k \geq 1 .
$$

Looking at the first component of the above vector identity, we infer that

$$
\left|E_{\rho}^{k}\right|=\rho|\Omega| \quad \forall k \geq 1
$$

Now, considering the remaining components, we observe that

$$
\int_{\Omega} \frac{1}{\rho} \chi_{E_{\rho}^{k}} v_{j} d x=\frac{1}{\rho} \int_{E_{\rho}^{k}} v_{j} d x=\int_{\Omega} v_{j}(x) d x \quad \forall j \geq 1 .
$$

This implies that

$$
\lim _{k \rightarrow \infty} \int_{\Omega} \frac{1}{\rho} \chi_{E_{\rho}^{k}} v_{j} d x=\int_{\Omega} v_{j}(x) d x \quad \forall j \geq 1 .
$$

From the density of $\left\{v_{j}\right\}_{j=1}^{\infty}$ in $L^{1}(\Omega)$ we conclude that

$$
\lim _{k \rightarrow \infty} \int_{\Omega} \frac{1}{\rho} \chi_{E_{\rho}^{k}} v d x=\int_{\Omega} v(x) d x \quad \forall v \in L^{1}(\Omega) .
$$

Copyright (c) by SIAM. Unauthorized reproduction of this article is prohibited. 
This means that $\frac{1}{\rho} \chi_{E_{\rho}^{k}} \stackrel{*}{\rightarrow} 1$ in $L^{\infty}(\Omega)$ as $k \rightarrow \infty$. Therefore, since $h \in L^{\bar{p}}(\Omega)$, it holds that $\left(1-\frac{1}{\rho} \chi_{E_{\rho}^{k}}\right) h \rightarrow 0$ in $L^{\bar{p}}(\Omega)$. Due to the fact that $\bar{p}>\frac{n}{2}$, there exists some $1<q<\frac{n}{n-1}$ such that $L^{\bar{p}}(\Omega)$ is compactly embedded in $W^{1, q}(\Omega)^{*}$. Consequently, we have the strong convergence $\left(1-\frac{1}{\rho} \chi_{E_{\rho}^{k}}\right) h \rightarrow 0$ in $W^{1, q}(\Omega)^{*}$. Thus, we can select $E_{\rho}=E_{\rho}^{k}$ with some sufficiently large $k$, so that

$$
\left\|\left(1-\frac{1}{\rho} \chi_{E_{\rho}}\right) h\right\|_{W^{1, q}(\Omega)^{*}}<\rho .
$$

Now, we define

$$
u_{\rho}(x)= \begin{cases}u(x) & \text { if } x \in E_{\rho} \\ \bar{u}(x) & \text { otherwise. }\end{cases}
$$

The reader is referred to $[4,5,8,12,22]$ for some previous papers using this type of diffuse perturbations. Obviously, $u_{\rho}$ belongs to $\mathcal{U}_{a d}$. Let us denote by $y_{\rho}$ and $\bar{y}$ the states associated with $u_{\rho}$ and $\bar{u}$, respectively. We also set $z_{\rho}=\frac{1}{\rho}\left(y_{\rho}-\bar{y}\right)$. Subtracting the equations satisfied by $y_{\rho}$ and $\bar{y}$ and dividing by $\rho$, using the mean value theorem and definition (4.6), we get

$$
\begin{aligned}
A z_{\rho} & =\frac{1}{\rho}\left[f\left(x, y_{\rho}, u_{\rho}\right)-f(x, \bar{y}, \bar{u})\right] \\
& =\frac{1}{\rho}\left[f\left(x, y_{\rho}, u_{\rho}\right)-f\left(x, \bar{y}, u_{\rho}\right)\right]+\frac{1}{\rho}\left[f\left(x, \bar{y}, u_{\rho}\right)-f(x, \bar{y}, \bar{u})\right] \\
& =\frac{\partial f}{\partial y}\left(x, \bar{y}+\theta_{\rho}\left(y_{\rho}-\bar{y}\right), u_{\rho}\right) z_{\rho}+\frac{1}{\rho} \chi_{E_{\rho}}[f(x, \bar{y}, u)-f(x, \bar{y}, \bar{u})] .
\end{aligned}
$$

Since $u_{\rho} \rightarrow \bar{u}$ in $L^{\bar{p}}(\Omega)$ when $\rho \rightarrow 0$, we know by Theorem 2.1 that $y_{\rho} \rightarrow \bar{y}$ in $H^{1}(\Omega) \cap C^{0, \mu}(\bar{\Omega})$. Then, using again [18, section 3.14], we deduce from (4.5) that $z_{\rho} \rightarrow z$ in $H^{1}(\Omega) \cap C^{0, \mu}(\bar{\Omega})$, where $z$ is the solution of

$$
\left\{\begin{aligned}
A z & =\frac{\partial f}{\partial y}(x, \bar{y}, \bar{u}) z+[f(x, \bar{y}, u)-f(x, \bar{y}, \bar{u})] & & \text { in } \Omega, \\
\partial_{n_{A}} z & = & & \text { on } \Gamma .
\end{aligned}\right.
$$

Now we take into account that, due to (4.4), the next inequality holds,

$$
\left\|u_{\rho}-\bar{u}\right\|_{L^{p}(\Omega)}=\|u-\bar{u}\|_{L^{p}\left(E_{\rho}\right)} \leq(\beta-\alpha)\left|E_{\rho}\right|^{\frac{1}{p}}<(\beta-\alpha)(\rho|\Omega|)^{\frac{1}{p}} .
$$

It implies that $u_{\rho} \in B_{\varepsilon}(\bar{u})$ holds for every $\rho$ sufficiently small. Therefore, from the local optimality of $\bar{u}$ we obtain

$$
0 \leq \lim _{\rho \rightarrow 0} \frac{J\left(u_{\rho}\right)-J(\bar{u})}{\rho}=\lim _{\rho \rightarrow 0} \int_{\Omega} \frac{\partial L}{\partial y}\left(x, \bar{y}+\vartheta_{\rho}\left(y_{\rho}-\bar{y}\right)\right) z_{\rho} d x=\int_{\Omega} \frac{\partial L}{\partial y}(x, \bar{y}) z d x .
$$

Taking the adjoint state $\bar{\varphi}$ associated with $\bar{u}$ and using (4.7), we get from the above inequality that

$$
\begin{aligned}
0 & \leq \int_{\Omega} \frac{\partial L}{\partial y}(x, \bar{y}) z d x=\int_{\Omega}\left[A^{*} \bar{\varphi}-\frac{\partial f}{\partial y}(x, \bar{y}, \bar{u}) \bar{\varphi}\right] z d x \\
& =\int_{\Omega}\left[A z-\frac{\partial f}{\partial y}(x, \bar{y}, \bar{u}) z\right] \bar{\varphi} d x=\int_{\Omega}[f(x, \bar{y}, u)-f(x, \bar{y}, \bar{u})] \bar{\varphi} d x
\end{aligned}
$$

Copyright $@$ by SIAM. Unauthorized reproduction of this article is prohibited. 
This leads to

$$
\int_{\Omega} H(x, \bar{y}(x), \bar{\varphi}(x), \bar{u}(x)) d x \leq \int_{\Omega} H(x, \bar{y}(x), \bar{\varphi}(x), u(x)) d x .
$$

Since $u \in \mathcal{U}_{a d}$ was arbitrary, we conclude that

$$
\int_{\Omega} H(x, \bar{y}(x), \bar{\varphi}(x), \bar{u}(x)) d x=\min _{u \in \mathcal{U}_{a d}} \int_{\Omega} H(x, \bar{y}(x), \bar{\varphi}(x), u(x)) d x .
$$

Finally, by using the classical spike perturbations, we deduce (4.2) from (4.8); see $[1$, Proposition 4.6] for details.

Next we formulate the second-order necessary conditions for local optimality. To this end, we first observe that by the representation $(2.14)$ the functional $J^{\prime}(\bar{u})$ : $L^{\infty}(\Omega) \longrightarrow \mathbb{R}$ can be extended to a linear continuous form $J^{\prime}(\bar{u}): L^{2}(\Omega) \longrightarrow \mathbb{R}$. Now, we define the cone of critical directions

$C_{\bar{u}}=\left\{v \in L^{2}(\Omega): J^{\prime}(\bar{u}) v=0\right.$ and $v$ satisfies the sign conditions (4.9) below $\}$,

$$
v(x) \begin{cases}\geq 0 & \text { if } \bar{u}(x)=\alpha, \\ \leq 0 & \text { if } \bar{u}(x)=\beta\end{cases}
$$

We have the following well-known result; see, for instance, [9] for a proof.

TheOREm 4.2. Under Assumptions $1-3$, if $\bar{u}$ is a local solution of $(\mathrm{P})$ in the $L^{p}(\Omega)$ sense $(1 \leq p \leq \infty)$, then $J^{\prime \prime}(\bar{u}) v^{2} \geq 0 \forall v \in C_{\bar{u}}$.

5. Second-order sufficient optimality conditions for $\boldsymbol{L}^{\infty}$-local solutions. In this section, following [10] and [11], we are going to impose a structural assumption on $\frac{\partial H}{\partial u}(x, \bar{y}, \bar{u})$, where $\bar{u} \in \mathcal{U}_{a d}$ is a control satisfying the optimality condition (4.1).

Assumption 4. There exist three constants $K>0, \varepsilon_{0}>0$, and $\gamma \in(0,+\infty]$ such that

$$
\left|\left\{x \in \Omega:\left|\bar{\varphi}(x) \frac{\partial f}{\partial u}(x, \bar{y}(x), \bar{u}(x))\right|<\varepsilon\right\}\right| \leq K \varepsilon^{\gamma} \quad \forall \varepsilon \leq \varepsilon_{0} .
$$

Remark 5.1. It is obvious that $\bar{u}$ is a bang-bang control if it fulfills Assumption 4. Moreover, let us observe that (5.1) is satisfied with $\gamma=+\infty$ and some $\varepsilon_{0}>0$ if and only if there exists a number $\sigma>0$ such that

$$
\left|\left\{x \in \Omega:\left|\bar{\varphi}(x) \frac{\partial f}{\partial u}(x, \bar{y}(x), \bar{u}(x))\right|<\sigma\right\}\right|=0 .
$$

We have the following result.

Theorem 5.2. Suppose that Assumptions 1-4 hold and $\bar{u} \in \mathcal{U}_{\text {ad }}$ satisfies the variational inequality (4.1). Then, there exists a constant $\kappa>0$ such that

$$
J^{\prime}(\bar{u})(u-\bar{u}) \geq \kappa\|u-\bar{u}\|_{L^{1}(\Omega)}^{1+\frac{1}{\gamma}} \quad \forall u \in \mathcal{U}_{a d}
$$

with $\kappa=\frac{1}{2[2(\beta-\alpha) K]^{\frac{1}{\gamma}}}$.

Proof. By standard arguments we infer that (4.1) holds pointwise, i.e.,

Copyright $@$ by SIAM. Unauthorized reproduction of this article is prohibited. 


$$
\begin{aligned}
& \frac{\partial H}{\partial u}(x, \bar{y}(x), \bar{\varphi}(x), \bar{u}(x))(u(x)-\bar{u}(x)) \\
& \quad=\bar{\varphi}(x) \frac{\partial f}{\partial u}(x, \bar{y}(x), \bar{u}(x))(u(x)-\bar{u}(x)) \geq 0 \quad \forall u \in \mathcal{U}_{a d} \text { and for a.a. } x \in \Omega .
\end{aligned}
$$

Now, given $\varepsilon>0$, we define

$$
\Omega_{\varepsilon}=\left\{x \in \Omega:\left|\bar{\varphi}(x) \frac{\partial f}{\partial u}(x, \bar{y}(x), \bar{u}(x))\right| \geq \varepsilon\right\} .
$$

From (5.1) we know that

$$
\left|\Omega \backslash \Omega_{\varepsilon}\right| \leq K \varepsilon^{\gamma}
$$

hence, we have

$$
\begin{aligned}
J^{\prime}(\bar{u})(u-\bar{u}) & =\int_{\Omega} \bar{\varphi}(x) \frac{\partial f}{\partial u}(x, \bar{y}(x), \bar{u}(x))(u(x)-\bar{u}(x)) d x \\
& \geq \int_{\Omega_{\varepsilon}}\left|\bar{\varphi}(x) \frac{\partial f}{\partial u}(x, \bar{y}(x), \bar{u}(x))\right||u(x)-\bar{u}(x)| d x \geq \varepsilon\|u-\bar{u}\|_{L^{1}\left(\Omega_{\varepsilon}\right)} \\
& =\varepsilon\|u-\bar{u}\|_{L^{1}(\Omega)}-\varepsilon\|u-\bar{u}\|_{L^{1}\left(\Omega \backslash \Omega_{\varepsilon}\right)} \\
& \geq \varepsilon\|u-\bar{u}\|_{L^{1}(\Omega)}-\varepsilon(\beta-\alpha)\left|\Omega \backslash \Omega_{\varepsilon}\right| \geq \varepsilon\|u-\bar{u}\|_{L^{1}(\Omega)}-(\beta-\alpha) K \varepsilon^{1+\gamma} .
\end{aligned}
$$

Now, choosing

$$
\varepsilon=[2(\beta-\alpha) K]^{-\frac{1}{\gamma}}\|u-\bar{u}\|_{L^{1}(\Omega)}^{\frac{1}{\gamma}},
$$

we get

$$
J^{\prime}(\bar{u})(u-\bar{u}) \geq \frac{1}{2[2(\beta-\alpha) K]^{\frac{1}{\gamma}}}\|u-\bar{u}\|_{L^{1}(\Omega)}^{1+\frac{1}{\gamma}} .
$$

Theorem 5.3. Let Assumptions 1-4 and the condition $\gamma=+\infty$ be fulfilled. If $\bar{u} \in \mathcal{U}_{\text {ad }}$ satisfies (4.1), then there exists $\varepsilon>0$ such that

$$
J(\bar{u})+\frac{\kappa}{2}\|u-\bar{u}\|_{L^{1}(\Omega)} \leq J(u) \quad \forall u \in \mathcal{U}_{a d} \cap \bar{B}_{\varepsilon}(\bar{u}),
$$

where $\bar{B}_{\varepsilon}(\bar{u})$ is the closed ball in $L^{\infty}(\Omega)$ and $\kappa$ is taken from (5.2). Therefore, $\bar{u}$ is strictly locally optimal in the sense of $L^{\infty}(\Omega)$.

Proof. Let us take $u \in \mathcal{U}_{a d} \cap \bar{B}_{\varepsilon}(\bar{u})$ with $\varepsilon>0$ to be fixed later. We perform a second-order Taylor expansion of $J$ at $\bar{u}$ and insert the expression of $J^{\prime \prime}$ given in (2.15). Invoking the growth condition (5.2), in view of the assumptions (2.8), (2.2), and (2.5) we obtain that

$$
\begin{aligned}
J(u) & =J(\bar{u})+J^{\prime}(\bar{u})(u-\bar{u})+\frac{1}{2} J^{\prime \prime}(\bar{u}+\theta(u-\bar{u}))(u-\bar{u})^{2} \\
& \geq J(\bar{u})+\kappa\|u-\bar{u}\|_{L^{1}(\Omega)}-C_{\alpha, \beta}\|u-\bar{u}\|_{L^{2}(\Omega)}^{2} \\
& \geq J(\bar{u})+\left[\kappa-C_{\alpha, \beta}\|u-\bar{u}\|_{L^{\infty}(\Omega)}\right]\|u-\bar{u}\|_{L^{1}(\Omega)} \geq J(\bar{u})+\left[\kappa-C_{\alpha, \beta} \varepsilon\right]\|u-\bar{u}\|_{L^{1}(\Omega)}
\end{aligned}
$$

holds for some $\theta \in(0,1)$ and a constant $C_{\alpha, \beta}$ independent of $u$. Then, it is enough to take $\varepsilon \leq \frac{\kappa}{2 C_{\alpha, \beta}}$ to obtain (5.3).

The reader might be surprised that we admit the possibility of the value $\gamma=+\infty$. The next example shows that this is possible for our control problem, indeed.

Copyright $@$ by SIAM. Unauthorized reproduction of this article is prohibited. 
Example 5.4 (a local solution in the sense of $L^{\infty}(\Omega)$ ). We consider the optimal control problem

$$
\begin{aligned}
& \min \int_{\Omega}\left(y_{u}-y_{d}\right)^{2} d x \\
& \left\{\begin{aligned}
-\Delta y(x)+y(x) & =e^{-y(x)} u(x)^{2}+2 e^{u(x)-2}+e_{\Omega}(x) & & \text { in } \Omega, \\
\partial_{n} y & =0 & & \text { on } \Gamma,
\end{aligned}\right. \\
& -1 \leq u(x) \leq 1 \text { a.e. in } \Omega,
\end{aligned}
$$

posed in $\Omega=(0,2 \pi) \times(0,2 \pi)$. Let us introduce the subdomains $\Omega_{1}=(0, \pi) \times(0, \pi)$, $\Omega_{2}=(\pi, 2 \pi) \times(0, \pi), \Omega_{3}=(0, \pi) \times(\pi, 2 \pi), \Omega_{4}=(\pi, 2 \pi) \times(\pi, 2 \pi)$.

First, we select a state $\bar{y} \in H^{2}(\Omega)$ with homogeneous Neumann data and satisfying $\|\bar{y}\|_{C(\bar{\Omega})}<3$. For instance, $\bar{y}(x) \equiv 1$ or $\bar{y}(x)=\cos \left(x_{1}\right) \cos \left(x_{2}\right)$ fulfill these conditions. We will construct the example so that $\bar{y}$ is optimal. To this end, we also define the adjoint state $\bar{\varphi}(x)=-1$ and the following bang-bang control $\bar{u}(x)$ :

$$
\bar{u}(x)= \begin{cases}+1 & \text { in } \Omega_{1} \cup \Omega_{4}, \\ -1 & \text { in } \Omega_{2} \cup \Omega_{3}\end{cases}
$$

We will now fix $y_{d}$ and $e_{\Omega}$ such that the triplet $(\bar{y}, \bar{\varphi}, \bar{u})$ obeys the first-order optimality system. Inserting $\bar{u}$ and $\bar{y}$ in the state equation, we find

$$
e_{\Omega}=-\Delta \bar{y}+\bar{y}-e^{-\bar{y}}-2 e^{\bar{u}-2} .
$$

Inserting $\bar{\varphi}=-1$ in the adjoint equation (2.16), we obtain $-1=e^{-\bar{y}}+\bar{y}-y_{d}$, hence $y_{d}=1+\bar{y}+e^{-\bar{y}}$.

Next, we check the variational inequality (4.1) for $\bar{u}$. We find

$$
\left[\bar{\varphi} \frac{\partial f}{\partial u}(\cdot, \bar{y}, \bar{u})\right](x)=-\left(2 e^{-\bar{y}} \bar{u}+2 e^{\bar{u}-2}\right)(x)=\left\{\begin{aligned}
-2 e^{-\bar{y}(x)}-2 e^{-1} & \text { if } \bar{u}(x)=+1, \\
2 e^{-\bar{y}(x)}-2 e^{-3} & \text { if } \bar{u}(x)=-1 .
\end{aligned}\right.
$$

In $\Omega_{1} \cup \Omega_{4}$, we have fixed $\bar{u}=1$ and hence we need that $\bar{\varphi}(x) \frac{\partial f}{\partial u}(x, \bar{y}(x), \bar{u}(x)) \leq 0$ to satisfy the variational inequality. This, however, is an immediate consequence of $-2 e^{-\bar{y}(x)}-2 e^{-1} \leq 0$. In $\Omega_{2} \cup \Omega_{3}$, from $\bar{u}=-1$ we deduce that $\bar{\varphi}(x) \frac{\partial f}{\partial u}(x, \bar{y}(x),-1) \geq 0$ must hold, hence the inequality $2 e^{-\bar{y}(x)}-2 e^{-3} \geq 0$ is needed. This follows from our assumption $\|\bar{y}\|_{C(\bar{\Omega})}<3$. Therefore, $\bar{u}$ satisfies the first-order necessary optimality conditions.

Finally, we prove that $\bar{u}$ satisfies (5.1) with $\gamma=+\infty$. Let us set

$$
\sigma=2\left[e^{-\|\bar{y}\|_{C(\bar{\Omega})}}-e^{-3}\right]>0 .
$$

Then, for $\bar{u}(x)=+1$ we have

$$
\left|\bar{\varphi}(x) \frac{\partial f}{\partial u}(x, \bar{y}(x), \bar{u}(x))\right|=2\left[e^{-\bar{y}(x)}+e^{-1}\right] \geq 2\left[e^{-\|\bar{y}\|_{C(\bar{\Omega})}}+e^{-1}\right]>\sigma .
$$

If $\bar{u}(x)=-1$, we get

$$
\left|\bar{\varphi}(x) \frac{\partial f}{\partial u}(x, \bar{y}(x), \bar{u}(x))\right|=2\left[e^{-\bar{y}(x)}-e^{-3}\right] \geq 2\left[e^{-\|\bar{y}\|_{C(\bar{\Omega})}}-e^{-3}\right]=\sigma .
$$

Copyright (c) by SIAM. Unauthorized reproduction of this article is prohibited. 
Thus, we conclude that

$$
\left|\left\{x \in \Omega:\left|\bar{\varphi}(x) \frac{\partial f}{\partial u}(x, \bar{y}(x), \bar{u}(x))\right|<\sigma\right\}\right|=0 .
$$

Therefore, $\gamma=+\infty$ can be taken and Theorem 5.2 yields that $\bar{u}$ is a strict local minimum of $(\mathrm{P})$ in the $L^{\infty}(\Omega)$-sense.

6. Second-order sufficient optimality conditions for $L^{2}(\Omega)$-local solutions. The goal of this section is to provide sufficient conditions for $L^{2}(\Omega)$-local optimality of a feasible control $\bar{u} \in \mathcal{U}_{a d}$ that satisfies the first-order necessary optimality conditions. The following theorem is the main result of this section.

TheOREM 6.1. Let $\bar{u} \in \mathcal{U}_{\text {ad }}$ satisfy the first-order optimality conditions (4.1) along with its associated state $\bar{y}$ and the adjoint state $\bar{\varphi}$. Besides Assumptions 13 , we suppose that

$$
\exists \nu>0 \text { such that } \frac{\partial^{2} H}{\partial u^{2}}(x, \bar{y}(x), \bar{\varphi}(x), s) \geq \nu \quad \text { for a.a. } x \in \Omega, \forall s \in[\alpha, \beta] .
$$

1. Under the hypothesis that

$$
\text { assumption (5.1) is fulfilled with } \gamma>1 \text {, }
$$

there exists $\varepsilon>0$ such that

$$
J(\bar{u})+\frac{\kappa}{2}\|u-\bar{u}\|_{L^{1}(\Omega)}^{1+\frac{1}{\gamma}}+\frac{\nu}{8}\|u-\bar{u}\|_{L^{2}(\Omega)}^{2} \leq J(u) \quad \forall u \in \mathcal{U}_{a d} \cap \bar{B}_{\varepsilon}(\bar{u}) .
$$

Here and below, $\bar{B}_{\varepsilon}(\bar{u})$ denotes the closed ball of $L^{2}(\Omega)$ with radius $\varepsilon$ centered at zero, and $\kappa$ is given in (5.2).

2. Under the hypothesis

$$
J^{\prime \prime}(\bar{u}) v^{2}>0 \quad \forall v \in C_{\bar{u}} \backslash\{0\},
$$

there exist $\varepsilon>0$ and $\delta>0$ such that

$$
J(\bar{u})+\frac{\delta}{2}\|u-\bar{u}\|_{L^{2}(\Omega)}^{2} \leq J(u) \quad \forall u \in \mathcal{U}_{a d} \cap \bar{B}_{\varepsilon}(\bar{u}) .
$$

For the proof of this theorem we will use the following two lemmas.

Lemma 6.2. Let $(X, \Sigma, \mu)$ be a measure space with $\mu(X)<+\infty$. Suppose that $\left\{g_{k}\right\}_{k=1}^{\infty} \subset L^{\infty}(X)$ and $\left\{v_{k}\right\}_{k=1}^{\infty} \subset L^{2}(X)$ satisfy the assumptions

- $g_{k} \geq 0$ a.e. in $X, \forall k \geq 1,\left\{g_{k}\right\}_{k=1}^{\infty}$ is bounded in $L^{\infty}(X)$, and $g_{k} \rightarrow g$ in $L^{1}(X), k \rightarrow \infty$,

- $v_{k} \rightarrow v$ in $L^{2}(X), k \rightarrow \infty$.

Then, there holds the inequality

$$
\int_{X} g(x) v^{2}(x) d \mu(x) \leq \liminf _{k \rightarrow \infty} \int_{X} g_{k}(x) v_{k}^{2}(x) d \mu(x) .
$$

The reader is referred to [9, Lemma 3.5] for the proof.

Lemma 6.3. Under Assumptions 1-3 and given $p>\frac{n}{2}$, there exist constants $C_{1}$ and $C_{p}$ such that $\forall u, \bar{u} \in \mathcal{U}_{a d}$ the following inequalities hold:

Copyright $@$ by SIAM. Unauthorized reproduction of this article is prohibited. 


$$
\begin{aligned}
& \left\|y_{u}-\bar{y}\right\|_{L^{2}(\Omega)} \leq C_{1}\|u-\bar{u}\|_{L^{1}(\Omega)}, \\
& \left\|y_{u}-\bar{y}\right\|_{L^{\infty}(\Omega)} \leq C_{p}\|u-\bar{u}\|_{L^{p}(\Omega)}, \\
& \left\|\varphi_{u}-\bar{\varphi}\right\|_{L^{\infty}(\Omega)} \leq C_{p}\|u-\bar{u}\|_{L^{p}(\Omega)}, \\
& \left\|z_{u, v}\right\|_{L^{2}(\Omega)} \leq C_{1}\|v\|_{L^{1}(\Omega)} \quad \forall v \in L^{1}(\Omega),
\end{aligned}
$$

where $y_{u}$ and $\bar{y}, \varphi_{u}$ and $\bar{\varphi}$ are the states and adjoint states associated with $u$ and $\bar{u}$, respectively. Moreover, we denote $z_{u, v}=G^{\prime}(u) v$.

Proof. From (2.8) we deduce the existence of a constant $M$ such that

$$
\left\|y_{u}\right\|_{C(\bar{\Omega})}+\|u\|_{L^{\infty}(\Omega)} \leq M \quad \forall u \in \mathcal{U}_{a d} .
$$

Subtracting the equations satisfied by $y_{u}$ and $\bar{y}$, we obtain with the mean value theorem

$$
\begin{aligned}
A\left(y_{u}-\bar{y}\right) & =\left[f\left(x, y_{u}, \bar{u}\right)-f(x, \bar{y}, \bar{u})\right]+\left[f\left(x, y_{u}, u\right)-f\left(x, y_{u}, \bar{u}\right)\right] \\
& =\frac{\partial f}{\partial y}\left(x, \bar{y}+\theta\left(y_{u}-\bar{y}\right), \bar{u}\right)\left(y_{u}-\bar{y}\right)+\left[f\left(x, y_{u}, u\right)-f\left(x, y_{u}, \bar{u}\right)\right] .
\end{aligned}
$$

Using the well-known estimates for linear systems, assumption (2.2), and the boundedness of $\mathcal{U}_{a d}$ in $L^{\infty}(Q)$, we infer

$$
\left\|y_{u}-\bar{y}\right\|_{L^{2}(\Omega)} \leq C\left\|f\left(x, y_{u}, u\right)-f\left(x, y_{u}, \bar{u}\right)\right\|_{L^{1}(\Omega)} \leq C C_{f, M}\|u-\bar{u}\|_{L^{1}(\Omega)},
$$

which implies (6.7).

To prove (6.8) we use again the equation satisfied by $y_{u}-\bar{y}$, the classical $L^{\infty}(\Omega)$ estimates for linear systems (see [23]), and again (2.2) to obtain

$$
\left\|y_{u}-\bar{y}\right\|_{L^{\infty}(\Omega)} \leq C(p)\left\|f\left(x, y_{u}, u\right)-f\left(x, y_{u}, \bar{u}\right)\right\|_{L^{p}(\Omega)} \leq C(p) C_{f, M}\|u-\bar{u}\|_{L^{p}(\Omega)},
$$

which proves (6.8).

To prove (6.9) we subtract the equations satisfied by $\varphi_{u}$ and $\bar{\varphi}$ to get

$$
\begin{aligned}
& A^{*}\left(\varphi_{u}-\bar{\varphi}\right)=\frac{\partial f}{\partial y}\left(x, y_{u}, u\right) \varphi_{u}-\frac{\partial f}{\partial y}(x, \bar{y}, \bar{u}) \bar{\varphi}+\frac{\partial L}{\partial y}\left(x, y_{u}\right)-\frac{\partial L}{\partial y}(x, \bar{y}) \\
& =\frac{\partial f}{\partial y}(x, \bar{y}, \bar{u})\left(\varphi_{u}-\bar{\varphi}\right)+\left[\frac{\partial f}{\partial y}\left(x, y_{u}, u\right)-\frac{\partial f}{\partial y}(x, \bar{y}, \bar{u})\right] \varphi_{u}+\left[\frac{\partial L}{\partial y}\left(x, y_{u}\right)-\frac{\partial L}{\partial y}(x, \bar{y})\right] .
\end{aligned}
$$

Using again the classical $L^{\infty}(\Omega)$-estimates, (2.2), (2.5), and (2.17), we deduce with (6.8)

$$
\begin{aligned}
& \left\|\varphi_{u}-\bar{\varphi}\right\|_{L^{\infty}(\Omega)} \\
& \quad \leq C^{\prime}(p)\left[C_{f, M} M^{*}\left(\left\|y_{u}-\bar{y}\right\|_{L^{p}(\Omega)}+\|u-\bar{u}\|_{L^{p}(\Omega)}\right)+C_{L, M}\left\|y_{u}-\bar{y}\right\|_{L^{p}(\Omega)}\right] \\
& \quad \leq C^{\prime \prime}(p)\|u-\bar{u}\|_{L^{p}(\Omega)} .
\end{aligned}
$$

Finally, (6.10) is an immediate consequence of (2.12) and (2.2).

Proof of Theorem 6.1. The proof is split into two parts.

Proof under assumption (6.2). Let $u \in \mathcal{U}_{a d} \cap \bar{B}_{\varepsilon}(\bar{u})$, where $\bar{B}_{\varepsilon}(\bar{u})$ is an $L^{2}(\Omega)$ closed ball around $\bar{u}$ with $\varepsilon>0$ to be fixed later. By a Taylor expansion, we get with $(5.2)$

Copyright (C) by SIAM. Unauthorized reproduction of this article is prohibited. 


$$
\begin{aligned}
J(u)= & J(\bar{u})+J^{\prime}(\bar{u})(u-\bar{u})+\frac{1}{2} J^{\prime \prime}(\bar{u}+\theta(u-\bar{u}))(u-\bar{u})^{2} \\
\geq & J(\bar{u})+\kappa\|u-\bar{u}\|_{L^{1}(\Omega)}^{1+\frac{1}{\gamma}}+\frac{1}{2} \int_{\Omega}\left\{\frac{\partial^{2} H}{\partial y^{2}}\left(x, y_{\theta}, \varphi_{\theta}, u_{\theta}\right) z_{\theta, u-\bar{u}}^{2}\right. \\
& \left.+2 \frac{\partial^{2} H}{\partial y \partial u}\left(x, y_{\theta}, \varphi_{\theta}, u_{\theta}\right) z_{\theta, u-\bar{u}}(u-\bar{u})+\frac{\partial^{2} H}{\partial u^{2}}\left(x, y_{\theta}, \varphi_{\theta}, u_{\theta}\right)(u-\bar{u})^{2}\right\} d x,
\end{aligned}
$$

where $u_{\theta}=\bar{u}+\theta(u-\bar{u})$ and $y_{\theta}$ and $\varphi_{\theta}$ denote the state and adjoint state associated with $u_{\theta}$. Moreover, $z_{\theta, u-\bar{u}}$ stands for $G^{\prime}\left(u_{\theta}\right)(u-\bar{u})$. Now, with (6.10), (6.11), (2.2), (2.5), and Young's inequality we infer

$$
\begin{aligned}
J(u) \geq & J(\bar{u})+\kappa\|u-\bar{u}\|_{L^{1}(\Omega)}^{1+\frac{1}{\gamma}}-C\|u-\bar{u}\|_{L^{1}(\Omega)}^{2}-\frac{\nu}{8}\|u-\bar{u}\|_{L^{2}(\Omega)}^{2} \\
& +\frac{1}{2} \int_{\Omega} \varphi_{\theta} \frac{\partial^{2} f}{\partial u^{2}}\left(x, y_{\theta}, u_{\theta}\right)(u-\bar{u})^{2} d x .
\end{aligned}
$$

Let us estimate the last integral in (6.12). First we write

$$
\begin{aligned}
\int_{\Omega} \varphi_{\theta} & \frac{\partial^{2} f}{\partial u^{2}}\left(x, y_{\theta}, u_{\theta}\right)(u-\bar{u})^{2} d x=\int_{\Omega}\left(\varphi_{\theta}-\bar{\varphi}\right) \frac{\partial^{2} f}{\partial u^{2}}\left(x, y_{\theta}, u_{\theta}\right)(u-\bar{u})^{2} d x \\
& +\int_{\Omega} \bar{\varphi}\left[\frac{\partial^{2} f}{\partial u^{2}}\left(x, y_{\theta}, u_{\theta}\right)-\frac{\partial^{2} f}{\partial u^{2}}\left(x, \bar{y}, u_{\theta}\right)\right](u-\bar{u})^{2} d x+\int_{\Omega} \bar{\varphi} \frac{\partial^{2} f}{\partial u^{2}}\left(x, \bar{y}, u_{\theta}\right)(u-\bar{u})^{2} d x \\
\geq & -\left\|\varphi_{\theta}-\bar{\varphi}\right\|_{L^{\infty}(\Omega)} C_{f, M}\|u-\bar{u}\|_{L^{2}(\Omega)}^{2} \\
& -M^{*}\left\|\frac{\partial^{2} f}{\partial u^{2}}\left(x, y_{\theta}, u_{\theta}\right)-\frac{\partial^{2} f}{\partial u^{2}}\left(x, \bar{y}, u_{\theta}\right)\right\|_{L^{\infty}(\Omega)}\|u-\bar{u}\|_{L^{2}(\Omega)}^{2}+\nu\|u-\bar{u}\|_{L^{2}(\Omega)}^{2},
\end{aligned}
$$

where we have used (6.11), (2.2), (2.17), and (6.1). Now, applying (6.8)-(6.9) with $p=2$, and (2.3) we get for sufficiently small $\varepsilon>0$ and $\|u-\bar{u}\|_{L^{2}(\Omega)}<\varepsilon$ the inequality

$$
\int_{\Omega} \varphi_{\theta} \frac{\partial^{2} f}{\partial u^{2}}\left(x, y_{\theta}, u_{\theta}\right)(u-\bar{u})^{2} d x \geq \frac{\nu}{2}\|u-\bar{u}\|_{L^{2}(\Omega)}^{2} .
$$

Now, we come back to (6.12) and observe that for $\gamma>1$

$$
\kappa\|u-\bar{u}\|_{L^{1}(\Omega)}^{1+\frac{1}{\gamma}}-C\|u-\bar{u}\|_{L^{1}(\Omega)}^{2}=\|u-\bar{u}\|_{L^{1}(\Omega)}^{1+\frac{1}{\gamma}}\left[\kappa-C\|u-\bar{u}\|_{L^{1}(\Omega)}^{1-\frac{1}{\gamma}}\right] \geq \frac{\kappa}{2}\|u-\bar{u}\|_{L^{1}(\Omega)}^{1+\frac{1}{\gamma}}
$$

if $\varepsilon$ is chosen such that

$$
C\|u-\bar{u}\|_{L^{1}(\Omega)}^{1-\frac{1}{\gamma}} \leq C|\Omega|^{\frac{1}{2}-\frac{1}{2 \gamma}}\|u-\bar{u}\|_{L^{2}(\Omega)}^{1-\frac{1}{\gamma}} \leq C|\Omega|^{\frac{1}{2}-\frac{1}{2 \gamma}} \varepsilon^{1-\frac{1}{\gamma}} \leq \frac{\kappa}{2} .
$$

Now (6.12) and (6.13) lead to (6.6).

Proof under assumption (6.4). Here we proceed by contradiction and assume the existence of a sequence $\left\{u_{k}\right\}_{k=1}^{\infty} \subset \mathcal{U}_{a d}$ such that

$$
\left\|u_{k}-\bar{u}\right\|_{L^{2}(\Omega)}<\frac{1}{k} \text { and } \quad J\left(u_{k}\right)<J(\bar{u})+\frac{1}{2 k}\left\|u_{k}-\bar{u}\right\|_{L^{2}(\Omega)}^{2} \quad \forall k \geq 1 .
$$

Let us define

$$
\rho_{k}=\left\|u_{k}-\bar{u}\right\|_{L^{2}(\Omega)} \quad \text { and } \quad v_{k}=\frac{1}{\rho_{k}}\left(u_{k}-\bar{u}\right) \text { for } k \geq 1 .
$$

Copyright $@$ by SIAM. Unauthorized reproduction of this article is prohibited. 
Since $\left\|v_{k}\right\|_{L^{2}(\Omega)}=1$, we can take a subsequence, denoted in the same way, such that $v_{k} \rightarrow v$ in $L^{2}(\Omega)$. Let us prove that $v \in C_{\bar{u}}$. Since the set of functions of $L^{2}(\Omega)$ satisfying (4.9) is convex and closed, and since every $v_{k}$ obviously satisfies (4.9), then $v$ also satisfies it. It remains to prove that $J^{\prime}(\bar{u}) v=0$. From the optimality conditions (4.1) we deduce

$$
J^{\prime}(\bar{u}) v=\lim _{k \rightarrow \infty} J^{\prime}(\bar{u}) v_{k}=\lim _{k \rightarrow \infty} \frac{1}{\rho_{k}} \int_{\Omega} \frac{\partial H}{\partial u}(x, \bar{y}, \bar{\varphi}, \bar{u})\left(u_{k}-\bar{u}\right) \geq 0 .
$$

To derive the converse inequality, we use (6.14)

$$
\begin{aligned}
\frac{\rho_{k}^{2}}{2 k} & =\frac{1}{2 k}\left\|u_{k}-\bar{u}\right\|_{L^{2}(\Omega)}^{2}>J\left(u_{k}\right)-J(\bar{u}) \\
& =J^{\prime}\left(\bar{u}+\theta_{k}\left(u_{k}-\bar{u}\right)\right)\left(u_{k}-\bar{u}\right)=\rho_{k} J^{\prime}\left(\bar{u}+\theta_{k}\left(u_{k}-\bar{u}\right)\right) v_{k} .
\end{aligned}
$$

Hence, we have

$$
\int_{\Omega} \varphi_{\theta_{k}} \frac{\partial f}{\partial u}\left(x, y_{\theta_{k}}, u_{\theta_{k}}\right) v_{k} d x=J^{\prime}\left(u_{\theta_{k}}\right) v_{k} \leq \frac{\rho_{k}}{2 k} \quad \forall k \geq 1,
$$

where $u_{\theta_{k}}=\bar{u}+\theta_{k}\left(u_{k}-\bar{u}\right)$ and $y_{\theta_{k}}$ and $\varphi_{\theta_{k}}$ are the associated state and adjoint state, respectively. Since $\left\|u_{\theta_{k}}-\bar{u}\right\|_{L^{2}(\Omega)} \leq\left\|u_{k}-\bar{u}\right\|_{L^{2}(\Omega)}<\frac{1}{k} \rightarrow 0$, it is easy to confirm with the aid of Theorem 2.1 that

$$
\varphi_{\theta_{k}} \frac{\partial f}{\partial u}\left(x, y_{\theta_{k}}, u_{\theta_{k}}\right) \longrightarrow \bar{\varphi} \frac{\partial f}{\partial u}(x, \bar{y}, \bar{u}) \text { in } L^{2}(\Omega) .
$$

Then, we can pass to the limit in (6.15) and deduce the desired converse inequality $J^{\prime}(\bar{u}) v \leq 0$, thus $J^{\prime}(\bar{u}) v=0$ and hence $v \in C_{\bar{u}}$.

Using again (6.14) and (4.1), and performing a second-order expansion, we obtain

$$
\begin{aligned}
\frac{\rho_{k}^{2}}{2 k}>J\left(u_{k}\right)-J(\bar{u})=J^{\prime}(\bar{u})\left(u_{k}-\bar{u}\right)+\frac{1}{2} J^{\prime \prime}\left(\bar{u}+\vartheta_{k}\left(u_{k}-\bar{u}\right)\right)\left(u_{k}-\bar{u}\right)^{2} \\
\geq \frac{1}{2} J^{\prime \prime}\left(\bar{u}+\vartheta_{k}\left(u_{k}-\bar{u}\right)\right)\left(u_{k}-\bar{u}\right)^{2}=\frac{\rho_{k}^{2}}{2} J^{\prime \prime}\left(\bar{u}+\vartheta_{k}\left(u_{k}-\bar{u}\right)\right) v_{k}^{2} .
\end{aligned}
$$

Dividing the above expression by $\frac{\rho_{k}^{2}}{2}$ we infer

$$
J^{\prime \prime}\left(\bar{u}+\vartheta_{k}\left(u_{k}-\bar{u}\right)\right) v_{k}^{2}<\frac{1}{k} \quad \forall k \geq 1 .
$$

Our next goal is to pass to the limit in (6.16) and to deduce that $J^{\prime \prime}(\bar{u}) v^{2} \leq 0$. Let us recall that according to (2.15) and the definition of the Hamiltonian we have

$$
\begin{aligned}
& J^{\prime \prime}(u) v^{2} \\
& =\int_{\Omega}\left\{\frac{\partial^{2} H}{\partial y^{2}}\left(x, y_{u}, \varphi_{u}, u\right) z_{u, v}^{2}+2 \frac{\partial^{2} H}{\partial y \partial u}\left(x, y_{u}, \varphi_{u}, u\right) z_{u, v} v+\frac{\partial^{2} H}{\partial u^{2}}\left(x, y_{u}, \varphi_{u}, u\right) v^{2}\right\} d x,
\end{aligned}
$$

where $z_{u, v}=G^{\prime}(u) v$. Let us set $u_{\vartheta_{k}}=\bar{u}+\vartheta_{k}\left(u_{k}-\bar{u}\right)$ and denote by $y_{\vartheta_{k}}$ and $\varphi_{\vartheta_{k}}$ its associated state and adjoint state, respectively.

From the convergence $u_{\vartheta_{k}} \rightarrow \bar{u}$ in $L^{2}(\Omega)$ and Theorem 2.1, we obtain that $y_{\vartheta_{k}} \rightarrow \bar{y}$ and $\varphi_{\vartheta_{k}} \rightarrow \bar{\varphi}$ in $H^{1}(\Omega) \cap C^{0, \mu}(\bar{\Omega})$. Moreover, the weak convergence $v_{k} \rightarrow v$ in $L^{2}(\Omega)$ yields the strong convergence $z_{\vartheta_{k}, v_{k}}=G^{\prime}\left(u_{\vartheta_{k}}\right) v_{k} \rightarrow z_{v}=G^{\prime}(\bar{u}) v$ in $L^{2}(\Omega)$. Additionally, we have that the sequences $\left\{\frac{\partial^{2} H}{\partial y^{2}}\left(x, y_{\vartheta_{k}}, \varphi_{\vartheta_{k}}, u_{\vartheta_{k}}\right)\right\}_{k=1}^{\infty}$ and 
$\left\{\frac{\partial^{2} H}{\partial y \partial u}\left(x, y_{\vartheta_{k}}, \varphi_{\vartheta_{k}}, u_{\vartheta_{k}}\right)\right\}_{k=1}^{\infty}$ are bounded in $L^{\infty}(\Omega)$ and converge to $\frac{\partial^{2} H}{\partial y^{2}}(x, \bar{y}, \bar{\varphi}, \bar{u})$ and $\frac{\partial^{2} H}{\partial y \partial u}(x, \bar{y}, \bar{\varphi}, \bar{u})$, respectively, in $L^{p}(\Omega)$ for every $p<\infty$. Then, we have

$$
\begin{aligned}
& \lim _{k \rightarrow \infty} \int_{\Omega}\left\{\frac{\partial^{2} H}{\partial y^{2}}\left(x, y_{\vartheta_{k}}, \varphi_{\vartheta_{k}}, u_{\vartheta_{k}}\right) z_{\vartheta_{k}, v_{k}}^{2}+2 \frac{\partial^{2} H}{\partial y \partial u}\left(x, y_{\vartheta_{k}}, \varphi_{\vartheta_{k}}, u_{\vartheta_{k}}\right) z_{\vartheta_{k}, v_{k}} v_{k}\right\} d x \\
& =\int_{\Omega}\left\{\frac{\partial^{2} H}{\partial y^{2}}(x, \bar{y}, \bar{\varphi}, \bar{u}) z_{v}^{2}+2 \frac{\partial^{2} H}{\partial y \partial u}(x, \bar{y}, \bar{\varphi}, \bar{u}) z_{v} v\right\} d x .
\end{aligned}
$$

Indeed, the first limit follows by applying Lebesgue's dominated convergence theorem. For the second limit it is enough to observe that $\frac{\partial^{2} H}{\partial y \partial u}\left(x, y_{\vartheta_{k}}, \varphi_{\vartheta_{k}}, u_{\vartheta_{k}}\right) z_{\vartheta_{k}, v_{k}} \rightarrow$ $\frac{\partial^{2} H}{\partial y \partial u}(x, \bar{y}, \bar{\varphi}, \bar{u}) z_{v}$ in $L^{2}(\Omega)$ and $v_{k} \rightarrow v$ in $L^{2}(\Omega)$.

Due to this convergence, to pass to the limit in (6.16) it remains to analyze the sequence $\left\{\frac{\partial^{2} H}{\partial u^{2}}\left(x, y_{\vartheta_{k}}, \varphi_{\vartheta_{k}}, u_{\vartheta_{k}}\right) v_{k}^{2}\right\}_{k=1}^{\infty}$. We proceed as follows. First, we write

$$
\begin{aligned}
& \frac{\partial^{2} H}{\partial u^{2}}\left(x, y_{\vartheta_{k}}, \varphi_{\vartheta_{k}}, u_{\vartheta_{k}}\right)=\left(\varphi_{\vartheta_{k}}-\bar{\varphi}\right) \frac{\partial^{2} f}{\partial u^{2}}\left(x, y_{\vartheta_{k}}, u_{\vartheta_{k}}\right) \\
& \quad+\bar{\varphi}\left[\frac{\partial^{2} f}{\partial u^{2}}\left(x, y_{\vartheta_{k}}, u_{\vartheta_{k}}\right)-\frac{\partial^{2} f}{\partial u^{2}}\left(x, \bar{y}, u_{\vartheta_{k}}\right)\right]+\bar{\varphi} \frac{\partial^{2} f}{\partial u^{2}}\left(x, \bar{y}, u_{\vartheta_{k}}\right)=I_{k, 1}+I_{k, 2}+I_{k, 3} .
\end{aligned}
$$

Using (6.11), (2.2), and (6.9) we get

$$
\lim _{k \rightarrow \infty}\left|I_{k, 1}\right| \leq \lim _{k \rightarrow \infty}\left\|\varphi_{\vartheta_{k}}-\bar{\varphi}\right\|_{L^{\infty}(\Omega)} C_{f, M}=0 .
$$

Now, given $\varepsilon>0$ arbitrary, from (2.3) and (6.8) we deduce the existence of $k_{\varepsilon}$ such that $\left|I_{k, 2}\right| \leq\|\bar{\varphi}\|_{L^{\infty}(\Omega)} \varepsilon \forall k \geq k_{\varepsilon}$. This yields

$$
\lim _{k \rightarrow \infty}\left|I_{k, 2}\right|=0 .
$$

Finally, from Lemma 6.2 we infer with (6.1)

$$
\int_{\Omega} \bar{\varphi} \frac{\partial^{2} f}{\partial u^{2}}(x, \bar{y}, \bar{u}) v^{2} d x \leq \liminf _{k \rightarrow \infty} \int_{\Omega} \bar{\varphi} \frac{\partial^{2} f}{\partial u^{2}}\left(x, \bar{y}, u_{\vartheta_{k}}\right) v_{k}^{2} d x .
$$

Collecting the last three results we obtain

$$
\int_{\Omega} \frac{\partial^{2} H}{\partial u^{2}}(x, \bar{y}, \bar{\varphi}, \bar{u}) v^{2} d x \leq \liminf _{k \rightarrow \infty} \int_{\Omega} \frac{\partial^{2} H}{\partial u^{2}}\left(x, y_{\vartheta_{k}}, \varphi_{\vartheta_{k}}, u_{\vartheta_{k}}\right) v_{k}^{2} d x .
$$

This inequality, along with (6.16) and (6.17), implies the next desired inequality

$$
J^{\prime \prime}(\bar{u}) v^{2} \leq \liminf _{k \rightarrow \infty} J^{\prime \prime}\left(u_{\vartheta_{k}}\right) v_{k}^{2} \leq 0 .
$$

But, according to (6.4), this is possible only if $v=0$. Therefore, $v_{k} \rightarrow 0$ in $L^{2}(\Omega)$ and $z_{v_{k}} \rightarrow 0$ in $L^{2}(\Omega)$. Therefore,

$$
\lim _{k \rightarrow \infty} \int_{\Omega}\left\{\frac{\partial^{2} H}{\partial y^{2}}\left(x, y_{\vartheta_{k}}, \varphi_{\vartheta_{k}}, u_{\vartheta_{k}}\right) z_{v_{k}}^{2}+2 \frac{\partial^{2} H}{\partial y \partial u}\left(x, y_{\vartheta_{k}}, \varphi_{\vartheta_{k}}, u_{\vartheta_{k}}\right) z_{v_{k}} v_{k}\right\} d x=0
$$

holds. In addition, from the properties stated for $I_{k, i}, i=1,2,3$, using (6.1) and the fact that $\left\|v_{k}\right\|_{L^{2}(\Omega)}=1$ for every $k$, we conclude 


$$
\begin{aligned}
\nu & =\liminf _{k \rightarrow \infty} \nu\left\|v_{k}\right\|_{L^{2}(\Omega)}^{2} \leq \liminf _{k \rightarrow \infty} \int_{\Omega} \bar{\varphi} \frac{\partial^{2} f}{\partial u^{2}}\left(x, \bar{y}, u_{\vartheta_{k}}\right) v_{k}^{2} d x \\
& \leq \liminf _{k \rightarrow \infty} \int_{\Omega} \varphi_{\vartheta_{k}} \frac{\partial^{2} f}{\partial u^{2}}\left(x, y_{\vartheta_{k}}, u_{\vartheta_{k}}\right) v_{k}^{2} d x .
\end{aligned}
$$

In this way, we have proved that $\nu \leq \liminf _{k \rightarrow \infty} J^{\prime \prime}\left(u_{\vartheta_{k}}\right) v_{k}^{2} \leq 0$. This is a contradiction to $\nu>0$.

Next we present the example of a control problem, where Theorem 6.1 is applicable.

Example 6.4 (a local solution in the sense of $L^{2}(\Omega)$ ). We consider the optimal control problem

$$
\begin{aligned}
& \min \frac{1}{2} \int_{\Omega}\left(y_{u}-y_{d}\right)^{2} d x \\
& \left\{\begin{aligned}
-\Delta y(x)+y(x) & =e^{-y(x)} u(x)^{2}+e^{2 u(x)}+e_{\Omega}(x) & & \text { in } \Omega, \\
\partial_{n} y & =0 & & \text { on } \Gamma,
\end{aligned}\right. \\
& -1 \leq u(x) \leq 1 \text { a.e. in } \Omega .
\end{aligned}
$$

Here, $y_{d}$ and $e_{\Omega} \in L^{2}(\Omega)$ will be fixed below. Thanks to Theorem 3.3, an optimal control $\bar{u}$ with associated state $\bar{y}$ exists. The adjoint equation is

$$
\left\{\begin{aligned}
-\Delta \bar{\varphi}+\bar{\varphi} & =-e^{-\bar{y}} \bar{u}^{2} \bar{\varphi}+\bar{y}-y_{d} & & \text { in } \Omega \\
\partial_{n} \varphi & =0 & & \text { on } \Gamma .
\end{aligned}\right.
$$

We select

$$
y_{d}=\bar{y}-1-e^{-\bar{y}} \bar{u}^{2},
$$

then $\bar{\varphi} \equiv 1$ is the adjoint state associated with $\bar{u}$. The variational inequality (4.1) for $\bar{u}$ is

$$
\int_{\Omega} \bar{\varphi} \frac{\partial f}{\partial u}(\cdot, \bar{y}, \bar{u})(u-\bar{u}) d x \geq 0 \quad \text { if }-1 \leq u(x) \leq+1 \text { for a.a. } x \in \Omega .
$$

Thanks to $\bar{\varphi} \equiv 1$, we have with $f=e^{-y} u^{2}+e^{2 u}+e_{\Omega}$

$$
\bar{\varphi} \frac{\partial f}{\partial u}(\cdot, \bar{y}, \bar{u})=2 e^{-\bar{y}} \bar{u}+2 e^{2 \bar{u}}
$$

hence $\bar{u}$ has to solve the equation

$$
\bar{u}(x)=\operatorname{Proj}_{[-1,1]}\left(-e^{\bar{y}(x)+2 \bar{u}(x)}\right) .
$$

Let us fix $\bar{u}(x) \equiv-1$ and $\bar{y} \equiv+2$. Inserting $\bar{y}$ in the state equation, $e_{\Omega}$ can be fixed accordingly: $e_{\Omega}=2\left(1-e^{-2}\right)$.

We confirm now that $\bar{u}=-1$ is locally optimal in the sense of $L^{2}(\Omega)$. The second derivative of the reduced objective functional $J$ is

$$
\begin{aligned}
J^{\prime \prime}(\bar{u}) v^{2} & =\int_{\Omega}\left\{z_{v}^{2}+\bar{\varphi}\left(e^{-\bar{y}} \bar{u}^{2} z_{v}^{2}-4 \bar{u} e^{-\bar{y}} v z_{v}+\left(2 e^{-\bar{y}}+4 e^{2 \bar{u}}\right) v^{2}\right)\right\} d x \\
& =\int_{\Omega}\left\{z_{v}^{2}+2 e^{-2}\left[\frac{1}{2} z_{v}^{2}+2 v z_{v}+3 v^{2}\right]\right\} d x \\
& =\int_{\Omega}\left\{z_{v}^{2}+2 e^{-2}\left[-\frac{1}{2} z_{v}^{2}+\left(z_{v}+v\right)^{2}+2 v^{2}\right]\right\} d x \geq 4 e^{-2}\|v\|_{L^{2}(\Omega)}^{2} .
\end{aligned}
$$

Copyright $@$ by SIAM. Unauthorized reproduction of this article is prohibited. 
Moreover, $\frac{\partial^{2} H}{\partial u^{2}}(\cdot, \bar{y}, \bar{\varphi}, \bar{u})=6 e^{-2}>0$. Then, thanks to Theorem 6.1 , the control $\bar{u}$ is locally optimal in the $L^{2}(\Omega)$-sense.

7. Convergence of numerical approximations. In this section, we assume that $\Omega$ is a convex domain in $\mathbb{R}^{n}$ with $n=2$ or 3 . We also suppose that $\Omega$ is polygonal if $n=2$ or polyhedral if $n=3$. In addition to Assumptions 1-3, we also assume that $a_{i j} \in C^{0,1}(\bar{\Omega})$ for $1 \leq i, j \leq n$ and $f(\cdot, 0,0) \in L^{2}(\Omega)$. Under these hypotheses it is well known that the solution of (1.1) belongs to $H^{2}(\Omega)$ and that, with (2.8) and Assumption 2, we have

$$
\exists M_{\alpha, \beta}>0 \text { such that }\left\|y_{u}\right\|_{H^{2}(\Omega)} \leq M_{\alpha, \beta} \quad \forall u \in \mathcal{U}_{a d} .
$$

The reader is referred to [17, section 3.2] for this regularity result.

Now, to perform the discretization of the control problem $(\mathrm{P})$, we consider a quasi-uniform family of triangulations $\left\{\mathcal{T}_{h}\right\}_{h>0}$ of $\bar{\Omega}$; cf. [2, Definition (4.4.13)]. We denote by $N_{h}$ the number of nodes of $\mathcal{T}_{h}$. Associated with these triangulations, we consider the finite dimensional spaces

$$
Y_{h}=\left\{y_{h} \in C(\bar{\Omega}): y_{h \mid T} \in P_{1}(T) \forall T \in \mathcal{T}_{h}\right\}
$$

and

$$
\mathcal{U}_{h}=\left\{u_{h} \in L^{\infty}(\Omega): u_{h \mid T} \in P_{0}(T) \forall T \in \mathcal{T}_{h}\right\},
$$

where $P_{i}(T)$ denotes the space of polynomials in $T$ of degree $i$. We set $U_{\text {ad, } h}=\mathcal{U}_{h} \cap \mathcal{U}_{\text {ad }}$.

Now, we introduce the discrete version of (1.1) as follows: for $u \in L^{\infty}(\Omega), y_{h}(u)$ is the solution of the nonlinear system

$$
\left\{\begin{array}{l}
\text { Find } y_{h} \in Y_{h} \text { such that } \\
a\left(y_{h}, z_{h}\right)=\int_{\Omega} f\left(x, y_{h}(x), u\right) z_{h}(x) d x \quad \forall z_{h} \in Y_{h} .
\end{array}\right.
$$

Above, $a: H^{1}(\Omega) \times H^{1}(\Omega) \longrightarrow \mathbb{R}$ denotes the following bilinear form associated with the operator $A$ :

$$
a\left(y_{1}, y_{2}\right)=\int_{\Omega}\left(\sum_{i, j=1}^{n} a_{i j}(x) \partial_{x_{i}} y_{1} \partial_{x_{j}} y_{2}+a_{0}(x) y_{1} y_{2}\right) d x .
$$

The existence of a solution of (7.2) is proved by an easy application of Browder's fixed point theorem. The uniqueness follows from the monotonicity of $f$ and the coercivity of the bilinear form $a$.

We approximate problem (P) by the discretized optimal control problem

$$
\min _{u_{h} \in U_{\mathrm{ad}, h}} J_{h}\left(u_{h}\right):=\int_{\Omega} L\left(x, y_{h}\left(u_{h}\right)(x)\right) d x,
$$

where $y_{h}\left(u_{h}\right)$ is the solution of $(7.2)$ for $u=u_{h}$. For $\left(\mathcal{P}_{h}\right)$, we obtain the following convergence theorem.

TheOREM 7.1. For every $h>0$, the discrete control problem $\left(\mathcal{P}_{h}\right)$ has at least one solution $\bar{u}_{h}$. Let $\left\{\bar{y}_{h}\right\}_{h>0}$ be a family of discrete optimal states associated with optimal controls $\left\{\bar{u}_{h}\right\}_{h>0}$. Then, $\left\{\bar{y}_{h}\right\}_{h>0}$ is bounded in $H^{1}(\Omega) \cap C(\bar{\Omega})$. Moreover, if $h_{k} \searrow 0$ when $k \rightarrow \infty$ and $\left\{\bar{y}_{h_{k}}\right\}_{k=1}^{\infty}$ converges weakly to $\bar{y}$ in $H^{1}(\Omega)$, then there exists $\bar{u} \in \mathcal{U}_{\text {ad }}$, an optimal solution of $(\mathrm{P})$, such that $\bar{y}$ is its associated state. Moreover, the sequence $\left\{\bar{y}_{h_{k}}\right\}_{k=1}^{\infty}$ converges strongly to $\bar{y}$ in $H^{1}(\Omega)$. 
Proof. The existence of an optimal control for the discretized problem follows from the compactness of $U_{\mathrm{ad}, h}$ in $\mathcal{U}_{h}$ and the continuity of $J_{h}$. Let us prove the boundedness of the discrete optimal states $\left\{\bar{y}_{h}\right\}_{h>0}$ in $H^{1}(\Omega) \cap C(\bar{\Omega})$. We define $M=\max \{|\alpha|,|\beta|\}$. Then, with Assumption 2 and the mean value theorem, we get the existence of a constant $C_{f, M}$ such that

$$
|f(x, 0, u(x))| \leq|f(x, 0,0)|+C_{f, M} M \quad \forall u \in \mathcal{U}_{a d} .
$$

This leads to the inequality

$$
\| f\left(\cdot, 0, u(\cdot)\left\|_{L^{2}(\Omega)} \leq\right\| f(\cdot, 0,0) \|_{L^{2}(\Omega)}+C_{f, M} M|\Omega|^{1 / 2} \quad \forall u \in \mathcal{U}_{a d} .\right.
$$

Then, from [7, Theorem 2], (7.1), and (7.4) we infer the existence of constants $C_{i}$, $i=1,2$, such that

$$
\begin{aligned}
& \left\|y_{u}-y_{h}(u)\right\|_{H^{1}(\Omega)} \leq C_{1} h \quad \forall u \in \mathcal{U}_{a d}, \\
& \left\|y_{u}-y_{h}(u)\right\|_{C(\bar{\Omega})} \leq C_{2} h^{2-\frac{n}{2}} \quad \forall u \in \mathcal{U}_{a d},
\end{aligned}
$$

where $y_{u} \in H^{1}(\Omega) \cap C^{0, \mu}(\bar{\Omega})$ and $y_{h}(u) \in Y_{h}$ are the solutions of (1.1) and (7.2), respectively. In [7, Theorem 2] this is done for the Dirichlet problem, but the same proof is valid for our state equation with homogeneous Neumann condition.

Combining (2.8) with (7.5)-(7.6) we infer

$$
\begin{aligned}
& \left\|\bar{y}_{h}\right\|_{H^{1}(\Omega)}+\left\|\bar{y}_{h}\right\|_{C(\bar{\Omega})} \leq\left\|\bar{y}_{h}-y_{\bar{u}_{h}}\right\|_{H^{1}(\Omega)}+\left\|\bar{y}_{h}-y_{\bar{u}_{h}}\right\|_{C(\bar{\Omega})} \\
& \quad+\left\|y_{\bar{u}_{h}}\right\|_{H^{1}(\Omega)}+\left\|y_{\bar{u}_{h}}\right\|_{C(\bar{\Omega})} \leq C_{1} h+C_{2} h^{2-\frac{n}{2}}+C_{\alpha, \beta}\left(\|f(\cdot, 0,0)\|_{L^{2}(\Omega)}+1\right) .
\end{aligned}
$$

Hence, $\left\{\bar{y}_{h}\right\}_{h>0}$ is bounded in $H^{1}(\Omega) \cap C(\bar{\Omega})$ and we can select a sequence $\left\{\bar{y}_{h_{k}}\right\}_{k=1}^{\infty}$ with $h_{k} \searrow 0$ such that $\bar{y}_{h_{k}} \rightarrow \bar{y}$ in $H^{1}(\Omega)$. Let us prove that $\bar{y}$ is an optimal state for (P). From the compactness of the embedding $H^{1}(\Omega) \subset L^{2}(\Omega)$ we know that $\bar{y}_{h_{k}} \rightarrow \bar{y}$ strongly in $L^{2}(\Omega)$ when $k \rightarrow \infty$. Now, we argue similarly to the proof of Theorem 3.3. To this end, we introduce the multifunction

$$
F: \Omega \longrightarrow \mathcal{P}(\mathbb{R}), \quad F(x)=\{f(x, \bar{y}(x), s): s \in[\alpha, \beta]\} .
$$

Since $f$ is continuous with respect to the last variable, we know that $F(x)$ is a closed and bounded interval of $\mathbb{R}$ for almost all $x \in \Omega$. Now, we define

$$
S=\left\{g \in L^{2}(\Omega): g(x) \in F(x) \text { for a.a. } x \in \Omega\right\} .
$$

$S$ is a convex and closed subset of $L^{2}(\Omega)$. Setting $g_{k}(x)=f\left(x, \bar{y}(x), u_{h_{k}}(x)\right) \forall k \geq 1$, all functions $g_{k}$ belong to $S$. Moreover, taking

$$
M=\max _{h>0}\left\|\bar{y}_{h}\right\|_{C(\bar{\Omega})}+\max \{|\alpha|,|\beta|\}<\infty
$$

from (2.2) and the mean value theorem we infer that $\left|g_{k}(x)\right| \leq|f(x, 0,0)|+C_{f, M} 2 M$, hence the sequence $\left\{g_{k}\right\}_{k=1}^{\infty}$ is bounded in $L^{2}(\Omega)$. By taking a subsequence, denoted in the same way, we can assume that $g_{k} \rightarrow \bar{g}$ in $L^{2}(\Omega)$. Since $S$ is weakly closed in $L^{2}(\Omega)$, we infer that $\bar{g} \in S$. Using again the classical Filippov theorem (see [16] or [19]), we deduce the existence of a measurable function $\bar{u}: \Omega \longrightarrow[\alpha, \beta]$, i.e., $\bar{u} \in \mathcal{U}_{a d}$, such that $\bar{g}(x)=f(x, \bar{y}(x), \bar{u}(x))$ for almost all $x \in \Omega$. Let us prove that $\bar{y}$ is the state associated with the control $\bar{u}$. We take $z \in H^{1}(\Omega)$ arbitrarily and select $z_{h_{k}} \in Y_{h_{k}}$ 
such that $z_{h_{k}} \rightarrow z$ strongly in $H^{1}(\Omega)$ when $k \rightarrow \infty$. For instance, we can take $z_{h_{k}}$ as the $H^{1}(\Omega)$-projection of $z$ on $Y_{h_{k}}$. Now, from (7.2) we get

$$
a\left(\bar{y}_{h_{k}}, z_{h_{k}}\right)=\int_{\Omega} g_{h_{k}} z_{h_{k}} d x+\int_{\Omega}\left[f\left(x, \bar{y}_{h_{k}}, \bar{u}_{h_{k}}\right)-f\left(x, \bar{y}, \bar{u}_{h_{k}}\right)\right] z_{h_{k}} d x .
$$

Using again (2.2) we obtain

$$
\left\|f\left(x, \bar{y}_{h_{k}}, \bar{u}_{h_{k}}\right)-f\left(x, \bar{y}, \bar{u}_{h_{k}}\right)\right\|_{L^{2}(\Omega)} \leq C_{f, M}\left\|\bar{y}_{h_{k}}-\bar{y}\right\|_{L^{2}(\Omega)} \rightarrow 0, \text { when } k \rightarrow \infty .
$$

Hence, passing to the limit in (7.7), we deduce

$$
a(\bar{y}, z)=\int_{\Omega} f(x, \bar{y}(x), \bar{u}(x)) z(x) d x .
$$

Since $z$ is arbitrary in $H^{1}(\Omega)$, we conclude that $A \bar{y}=f(x, \bar{y}(x), \bar{u}(x))$ and, hence, $\bar{y}$ is the state associated with $\bar{u}$.

Moreover, we are able to prove the strong convergence of $\bar{y}_{h_{k}}$ in $H^{1}(\Omega)$ : taking $z_{h_{k}}=\bar{y}_{h_{k}}$ in (7.7) and using the convergences $g_{h_{k}} \rightarrow f(x, \bar{y}, \bar{u})$ and $\bar{y}_{h_{k}} \rightarrow \bar{y}$ weakly and strongly, respectively, in $L^{2}(\Omega)$ and the identity $A \bar{y}=f(x, \bar{y}, \bar{u})$, it follows that

$$
\lim _{k \rightarrow \infty} a\left(\bar{y}_{h_{k}}, \bar{y}_{h_{k}}\right)=\lim _{k \rightarrow \infty} \int_{\Omega} g_{h_{k}} \bar{y}_{h_{k}} d x=\int_{\Omega} f(x, \bar{y}, \bar{u}) \bar{y} d x=a(\bar{y}, \bar{y}) .
$$

The convergence $a\left(\bar{y}_{h_{k}}, \bar{y}_{h_{k}}\right) \rightarrow a(\bar{y}, \bar{y})$ along with the weak convergence $\bar{y}_{h_{k}} \rightarrow \bar{y}$ in $H^{1}(\Omega)$ implies that $\bar{y}_{h_{k}} \rightarrow \bar{y}$ strongly in $H^{1}(\Omega)$.

It remains to prove that $\bar{u}$ is a solution of $(\mathrm{P})$. To this aim, we take an arbitrary $u \in \mathcal{U}_{a d}$ with associated state $y_{u}$ (solution of (1.1)). Define $u_{h}$ as the $L^{2}$-projection of $u$ on $\mathcal{U}_{h}$. Since $\mathcal{U}_{h}$ is a space of piecewise constant functions, it is well known that $u_{h} \in U_{\mathrm{ad}, h}$ and $u_{h} \rightarrow u$ strongly in $L^{2}(\Omega)$. Let us denote by $y_{u_{h}}$ and $y_{h}$ the solutions of (1.1) and (7.2), respectively, corresponding to the control $u_{h}$. Then, with (2.9) and (7.5)-(7.6) we infer

$$
\begin{gathered}
\left\|y_{u}-y_{h}\right\|_{H^{1}(\Omega)}+\left\|y_{u}-y_{h}\right\|_{C(\bar{\Omega})} \leq\left\|y_{u}-y_{u_{h}}\right\|_{H^{1}(\Omega)}+\left\|y_{u}-y_{u_{h}}\right\|_{C(\bar{\Omega})} \\
+\left\|y_{u_{h}}-y_{h}\right\|_{H^{1}(\Omega)}+\left\|y_{u_{h}}-y_{h}\right\|_{C(\bar{\Omega})} \rightarrow 0 \quad \text { when } h \rightarrow 0 .
\end{gathered}
$$

Now, setting $\tilde{M}=\max _{h>0}\left\|\bar{y}_{h}\right\|_{C(\bar{\Omega})}$, we deduce with Assumption 3 that

$$
\left|L\left(x, \bar{y}_{h_{k}}(x)\right)\right| \leq|L(x, 0)|+\psi_{\tilde{M}}(x) \tilde{M} \quad \text { a.e. in } \Omega .
$$

Then, the Lebesgue's dominated convergence theorem implies that $J_{h_{k}}\left(\bar{u}_{h_{k}}\right) \rightarrow J(\bar{u})$ when $k \rightarrow \infty$. A similar argument shows that $J_{h_{k}}\left(u_{h_{k}}\right) \rightarrow J(u)$. Finally, recalling that $\bar{u}_{h_{k}}$ is a solution of $\left(\mathcal{P}_{h_{k}}\right)$, we get

$$
J(\bar{u})=\lim _{k \rightarrow \infty} J_{h_{k}}\left(\bar{u}_{h_{k}}\right) \leq \lim _{k \rightarrow \infty} J_{h_{k}}\left(u_{h_{k}}\right)=J(u) .
$$

Since $u$ was taken arbitrarily in $\mathcal{U}_{a d}$, we conclude that $\bar{u}$ is solution of $(\mathrm{P})$.

\section{REFERENCES}

[1] F. Bonnans and E. Casas, An extension of Pontryagin's principle for state-constrained optimal control of semilinear elliptic equations and variational inequalities, SIAM J. Control Optim., 33 (1995), pp. 274-298.

[2] S. C. Brenner and L. R. Scott, The Mathematical Theory of Finite Element Methods, 3rd ed., Texts in Appl. Math. 15, Springer, New York, 2008. 
[3] E. CASAS, Boundary control of semilinear elliptic equations with pointwise state constraints, SIAM J. Control Optim., 31 (1993), pp. 993-1006.

[4] E. CASAS, Pontryagin's principle for optimal control problems governed by semilinear elliptic equations, in International Conference on Control and Estimation of Distributed Parameter Systems: Nonlinear Phenomena, F. Kappel and K. Kunisch, eds., Int. Ser. Numer. Anal. 118, Birkhäuser, Basel, 1994, pp. 97-114.

[5] E. CASAS, Pontryagin's principle for state-constrained boundary control problems of semilinear parabolic equations, SIAM J. Control Optim., 35 (1997), pp. 1297-1327.

[6] E. Casas And L. A. Fernández, A Green's formula for quasilinear elliptic operators, J. Math. Anal. Appl., 142 (1989), pp. 62-72.

[7] E. Casas and M. Mateos, Uniform convergence of the FEM. Applications to state constrained control problems, Comput. Appl. Math., 21 (2002), pp. 67-100.

[8] E. Casas, J.-P. Raymond, And H. Zidani, Pontryagin's principle for local solutions of control problems with mixed control-state constraints, SIAM J. Control Optim., 39 (2000), pp. $1182-1203$.

[9] E. CASAS AND F. Tröltzsch, Second order analysis for optimal control problems: Improving results expected from from abstract theory, SIAM J. Optim., 22 (2012), pp. 261-279.

[10] E. Casas, D. Wachsmuth, and G. Wachsmuth, Sufficient second-order conditions for bangbang control problems, SIAM J. Control Optim., 55 (2017), pp. 3066-3090.

[11] E. Casas, D. Wachsmuth, and G. Wachsmuth, Second order analysis and numerical approximation for bang-bang bilinear control problems, SIAM J. Control Optim., 56 (2018), pp. 4203-4227.

[12] E. CaSas And J. Yong, Maximum principle for state-constrained optimal control problems governed by quasilinear elliptic equations, Differential Integral Equations, 8 (1995), pp. $1-18$.

[13] L. Cesari, Optimization-Theory and Applications: Problems with Ordinary Differential Equations, Appl. Math. (N. Y.) 17, Springer, New York, 1983.

[14] K. EPPLER, On the existence of optimal solutions for time-optimal semilinear parabolic boundary control problems, Z. Anal. Anwend., 12 (1993), pp. 153-169.

[15] K. Eppler And A. Unger, Boundary control of semilinear elliptic equations-existence of optimal solutions, Control Cybernet., 26 (1997), pp. 249-259.

[16] A. F. Filippov, On some topics in the theory of optimal regulation, Vestnik Moskov. Univ., Ser. Mat. Mekh. Astron. Fiz. Khim., (2) 1959, pp. 25-32 (in Russian).

[17] P. Grisvard, Elliptic Problems in Nonsmooth Domains, Pitman, Boston, 1985.

[18] O. A. Ladyzhenskaya and N. N. URaL'Tseva, Linear and Quasilinear Elliptic Equations. Academic Press, New York, 1968.

[19] J. MaCKi AND A. Strauss, Introduction to Optimal Control Theory, Springer-Verlag, Berlin, 1982.

[20] H. Maurer AND H. OBerle, Second order sufficient conditions for optimal control problems with free final time: The Riccati approach, SIAM J. Control Optim., 41 (2002), pp. 380-403.

[21] N. S. Papageorgiou, An existence theorem for a class of nonlinear elliptic optimal control problems, Comment. Math. Univ. Carolin., 32 (1991), pp. 273-279.

[22] J.-P. Raymond and H. Zidani, Pontryagin's principle for state-constrained control problems governed by parabolic equations with unbounded controls, SIAM J. Control Optim., 36 (1998), pp. 1853-1879.

[23] G. Stampacchia, Le problème de Dirichlet pour les équations elliptiques du second ordre à coefficients discontinus, Ann. Inst. Fourier (Grenoble), 15 (1965), pp. 189-258.

[24] F. TRÖLTZSCH, Lipschitz stability of solutions of linear-quadratic parabolic control problems with respect to perturbations, Dyn. Contin. Discrete Impuls. Syst., 7 (2000), pp. 289-306.

[25] F. Tröltzsch, Optimal Control of Partial Differential Equations: Theory, Methods and Applications, Grad. Stud. Math. 112, AMS, Providence, RI, 2010.

Copyright (c) by SIAM. Unauthorized reproduction of this article is prohibited. 